\title{
Output Constrained Lossy Source Coding With Limited Common Randomness
}

\author{
Naci Saldi, Student Member, IEEE, Tamás Linder, Fellow, IEEE, and Serdar Yüksel, Member, IEEE
}

\begin{abstract}
This paper studies a Shannon-theoretic version of the generalized distribution preserving quantization problem where a stationary and memoryless source is encoded subject to a distortion constraint and the additional requirement that the reproduction also be stationary and memoryless with a given distribution. The encoder and decoder are stochastic and assumed to have access to independent common randomness. Recent work has characterized the minimum achievable coding rate at a given distortion level when unlimited common randomness is available. Here, we consider the general case where the available common randomness may be rate limited. Our main result completely characterizes the set of achievable coding and common randomness rate pairs at any distortion level, thereby providing the optimal tradeoff between these two rate quantities. We also consider two variations of this problem where we investigate the effect of relaxing the strict output distribution constraint and the role of private randomness used by the decoder on the rate region. Our results have strong connections with Cuff's recent work on distributed channel synthesis. In particular, our achievability proof combines a coupling argument with the approach developed by Cuff, where instead of explicitly constructing the encoder-decoder pair, a joint distribution is constructed from which a desired encoder-decoder pair is established. We show, however, that for our problem, the separated solution of first finding an optimal channel and then synthesizing this channel results in a suboptimal rate region.
\end{abstract}

Index Terms-Lossy source coding, rate distortion, randomization, shared randomness, channel synthesis.

\section{INTRODUCTION}

I $\mathrm{N}$ THIS paper, we aim to characterize the achievable rate distortion region for the generalized distribution preserving randomized source coding problem, where the rate region measures both the coding rate and the rate of common randomness shared between the encoder and the decoder. To give a more precise definition of the problem, consider the communication system in Fig. 1.

The source block $X^{n}=\left(X_{1}, \ldots, X_{n}\right)$ consists of $n$ independent drawings of a random variable $X$ which takes values in a set $\mathbf{X}$ and has distribution $\mu$. The stochastic encoder takes the source and the common randomness, which is

Manuscript received November 20, 2014; revised June 24, 2015; accepted June 24, 2015. Date of publication June 29, 2015; date of current version August 14, 2015. This work was supported in part by the Natural Sciences and Engineering Research Council of Canada. This paper was presented at the 52nd Annual Allerton Conference on Communication, Control and Computing in 2014.

The authors are with the Department of Mathematics and Statistics, Queen's University, Kingston, ON K7L 3N6, Canada (e-mail: nsaldi@mast.queensu.ca; linder@mast.queensu.ca; yuksel@mast.queensu.ca).

Communicated by E. Tuncel, Associate Editor for Source Coding.

Color versions of one or more of the figures in this paper are available online at http://ieeexplore.ieee.org.

Digital Object Identifier 10.1109/TIT.2015.2450721

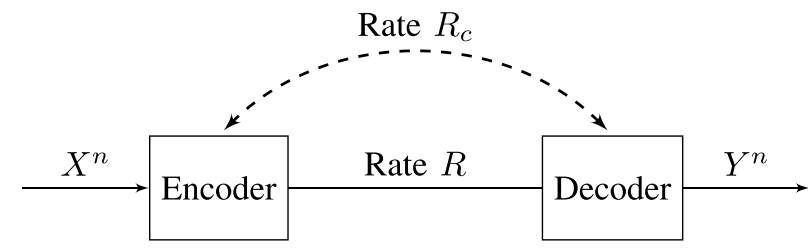

Fig. 1. Randomized source coding with limited common randomness.

available at rate $R_{c}$ bits per source symbol, as its inputs and produces an output at a rate $R$ bits per source symbol. Observing the output of the encoder and the common randomness, the decoder (stochastically) generates the output (reconstruction) which takes values from a reproduction alphabet $Y$. Here $X=Y$ is either a finite set or the real line. The common randomness is assumed to be independent of the source. As usual, the fidelity of the reconstruction is characterized by the expected distortion

$$
\mathbb{E}\left[\frac{1}{n} \sum_{i=1}^{n} \rho\left(X_{i}, Y_{i}\right)\right]
$$

where $\rho: \mathrm{X} \times \mathrm{Y} \rightarrow[0, \infty)$ is a distortion measure. However, unlike in the standard rate distortion problem, we require that the output $Y^{n}=\left(Y_{1}, \ldots, Y_{n}\right)$ be a sequence of independent and identically distributed (i.i.d.) random variables with $a$ given common distribution $\psi$.

For $D \geq 0$, a rate pair $\left(R, R_{c}\right)$ is said to be achievable at distortion level $D$ if, for any $\varepsilon>0$ and all $n$ large enough, there exists a system as in Fig. 1 with coding rate $R$ and common randomness rate $R_{c}$, such that the distortion of the system is less than $D+\varepsilon$ and the output distribution constraint for $Y^{n}$ holds. The main problem considered in this paper is finding the set of all achievable rate pairs, denoted by $\mathcal{R}(D)$.

The communication system depicted in Fig. 1 is a generalized version of a randomized quantizer (source code) where the encoder and decoder is usually assumed to have access to unlimited common randomization. Randomized (dithered) uniform quantizers were originally introduced in signal processing by Roberts [1], where he observed that adding random noise to an image signal before uniform quantization and subtracting the noise before reconstruction may result in perceptually more pleasing images. Versions of dithered uniform quantizers were analyzed by Schuchman [2] and Gray and Stockham [3]. Under certain conditions, dithering results in uniformly distributed quantization noise that is independent of the input [2], [3], which allows modeling the quantization process by an 
additive noise channel. Related entropy-coded dithered scalar and lattice quantizers have been extensively used in the information theoretic literature to construct robust lossy compression schemes with universal performance guarantees [4]-[7]. Akyol and Rose [8], [9], introduced a class of randomized nonuniform scalar quantizers obtained via applying companding to a dithered uniform quantizer. Recently Li et al. [10], [11] and Klejsa et al. [12] introduced and studied more general classes of randomized quantizers that are distribution-preserving, i.e., the quantizer output is restricted to have the same distribution as the source. The distribution-preserving property of these quantizers is reported to significantly improve the perceptual quality of the reconstruction in audio and video coding. Note that if in Fig. 1 we set the distribution $\mu$ of the $X_{i}$ to be equal to the distribution $\psi$ of the $Y_{i}$, we obtain a distribution-preserving quantizer.

In our recent work [13], [14] we studied a generalized version of distribution-preserving randomized quantization where the output is constrained to have a given distribution which may be different from the source distribution. The main focus there was to develop an abstract and completely general representation of finite-dimensional randomized quantization and to study the existence and structural properties of optimal generalized distribution preserving quantizers. Moreover, [14] also considered the asymptotic performance in the limit of infinite block length. In particular, a rate distortion theorem was obtained for stationary and memoryless sources under the assumption that the output must also be a stationary and memoryless process and common randomness (in the form of a random variable uniformly distributed on the unit interval $[0,1])$ is shared by the encoder and the decoder. This situation corresponds to formally setting $R_{c}=\infty$ in Fig. 1 . In particular, [14, Th. 7] showed for both finite and continuous source and reproduction alphabets that the set of achievable coding rates for unlimited common randomness $R_{c}=\infty$, denoted by $\mathcal{R}(D, \infty)$, is

$$
\mathcal{R}(D, \infty)=\left\{R \in \mathbb{R}: R \geq I(X ; Y), P_{X, Y} \in \mathcal{G}(D)\right\},
$$

where $\mathcal{G}(D)$ is the set of probability distributions $P_{X, Y}$ of $\mathrm{X} \times \mathrm{Y}$-valued random variables $(X, Y)$ defined as

$$
\mathcal{G}(D):=\left\{P_{X, Y}: P_{X}=\mu, P_{Y}=\psi, \mathbb{E}[\rho(X, Y)] \leq D\right\} .
$$

Thus the minimum coding rate at distortion $D$ is the so-called "minimum mutual information with constrained output $\psi$ " [15] given by

$$
I(\mu \| \psi, D):=\min \left\{I(X, Y): P_{X, Y} \in \mathcal{G}(D)\right\} .
$$

If $\mathcal{G}(D)$ is empty, we let $I(\mu \| \psi, D)=\infty$.

In this paper, we generalize the above rate distortion result by studying the optimal tradeoff between the coding rate $R$ and common randomness rate $R_{c}$ for the system in Fig. 1. In particular, we find a single-letter characterization of the entire achievable rate region $\mathcal{R}(D)$ of pairs $\left(R, R_{c}\right)$. Apart from the theoretical appeal of obtaining a computable characterization of the rate region via information theoretic quantities, this investigation is also motivated by the fact that the common randomness rate $R_{c}$ has a direct affect on the complexity of the system since each possible value of the common randomization picks a different (stochastic) encoder and decoder pair from a finite set whose size is proportional to $2^{n R_{c}}$. We also consider two variations of the problem, in which we investigate the effect of relaxing the strict output distribution constraint and the role of private randomness used by the decoder on the rate region. For both of these problems, we give the complete characterizations of the achievable rate pairs.

It is important to point out that the block diagram in Fig. 1 depicting the generalized distribution preserving quantization problem has the same structure as the system studied by Cuff [16], [17] to synthesize memoryless channels up to vanishing total variation error. Although many other problems in information theory share a similar representation, the connection with Cuff's work is more than formal. The distortion and output distribution constraints in our problem replaces the requirement in [17] that the joint distribution of the input $X^{n}$ and output $Y^{n}$ should arbitrarily well approximate (in total variation) the joint distribution obtained by feeding the input $X^{n}$ to a given memoryless channel. Using the main result [17, Th. II.1] one can obtain an inner bound, albeit a loose one, for our problem. A good part of our proof consists of tailoring Cuff's arguments in [17] to our setup to obtain a tight achievable rate region. Because of this, we will be adopting many of the notations used in [17]. We also note that unlike in the distributed channel synthesis problem in [17], our results also allow for continuous source and reproduction alphabets.

The rest of the paper is organized as follows. In Section II we formalize the problem and present the main result giving the rate region $\mathcal{R}(D)$. Section II-A discusses connections with Cuff's work on distributed channel synthesis. In Section III we investigate the extreme points of the rate region at $R_{c}=0$ and $R_{c}=\infty$. In Section IV we present computable inner bounds for double symmetric binary source and reproduction distributions under the Hamming distortion, and for Gaussian source and reproduction distributions under the squared error distortion. In Section V two variations of the original problem are formulated and the associated achievable rate regions are described. The proof of the main result is given in Section VI.

\section{A. Notation and Assumptions}

In this paper, $X$ denotes the input alphabet and $Y$ is the reconstruction (output) alphabet such that $X=Y$ is a finite set or $\mathrm{X}=\mathrm{Y}=\mathbb{R}$. We assume a distortion measure $\rho(x, y)=$ $d(x, y)^{p}$, where $d$ is the metric on $\mathrm{X}$. Here, $p>0$ when $\mathrm{X}$ is finite and $p=2$ when $\mathrm{X}=\mathbb{R}$, in which case we also assume that $d(x, y)=|x-y|$ (so that $\rho$ is the squared error) and that the source distribution $\mu$ and the desired output distribution $\psi$ have finite second moments. We note that we impose these restrictions on the distortion measure because in a key step of the achievability proof we need to invoke the triangle inequality. For the finite alphabet case, we let $\rho_{\max }:=\max _{x, y} \rho(x, y)$. For any positive real number $R$, we define $\left[2^{n R}\right]:=\left\{1, \ldots,\left\lceil 2^{n R}\right\rceil\right\}$, where $\left\lceil 2^{n R}\right\rceil$ is the smallest 
integer greater than or equal to $2^{n R}$. $\mathrm{V}^{n}$ will denote the $n$-fold Cartesian product of a set $\mathrm{V}$, the elements of which are $v^{n}=\left(v_{1}, \ldots, v_{n}\right), v_{i} \in \mathrm{V}, i=1, \ldots, n$. A similar convention also applies to a sequence of random variables which will be denoted by upper case letters. For any triple $(X, Y, U)$ of random variables or vectors, the notation $X-U-Y$ means that they form a Markov chain in this order. For any random vector $U^{n}$, the random measure $p_{U^{n}}$ denotes the empirical distribution of $U^{n}$. The notation $V \sim v$ means that random variable $V$ has distribution $v$. For any probability distribution $v$ on $\mathrm{V}, v^{n}$ denotes the $n$-fold product distribution $\underbrace{v \times \cdots \times v}_{n \text {-times }}$ on $\mathrm{V}^{n}$.

\section{Problem Statement and Main Result}

Let $\left\{X_{n}\right\}_{n \geq 1}$ be a stationary and memoryless source (sequence of i.i.d. random variables) with common distribution $\mu$ on source alphabet $\mathrm{X}$, and let $K$ be a random variable uniformly distributed over $\left[2^{n R_{c}}\right]$ which is independent of $X^{n}$. Here $K$ represents the common randomness that is shared between the encoder and the decoder.

For a positive integer $n$ and nonnegative numbers $R$ and $R_{C}$, a $\left(n, R, R_{c}\right)$ randomized source code is defined by an encoder $E=E_{J \mid X^{n}, K}$ and the decoder $F_{Y^{n} \mid J, K}$, where $E$ is a regular conditional probability (see [18]) on $\left[2^{n R}\right]$ given $\mathrm{X}^{n} \times\left[2^{n R_{c}}\right]$ and $F$ is a regular conditional probability on $\mathrm{Y}^{n}$ given $\left[2^{n R}\right] \times\left[2^{n R_{c}}\right]$. Hence, letting $J$ and $Y^{n}$ be the output of the encoder and the decoder, respectively, the joint distribution of $\left(K, X^{n}, J, Y^{n}\right)$ is given, in a somewhat informal notation, by

$$
\left(K, X^{n}, J, Y^{n}\right) \sim F_{Y^{n} \mid J, K} E_{J \mid X^{n}, K} P_{K} P_{X^{n}} .
$$

The distortion of the code is $\mathbb{E}\left[\rho_{n}\left(X^{n}, Y^{n}\right)\right]$, where $\rho_{n}\left(x^{n}, y^{n}\right):=\frac{1}{n} \sum_{i=1}^{n} \rho\left(x_{i}, y_{i}\right)$.

Definition 1: For any nonnegative real number $D$ and desired output distribution $\psi$, the pair $\left(R, R_{c}\right)$ is said to be $\psi$-achievable if, for any $\varepsilon>0$ and all sufficiently large $n$, there exists a randomized $\left(n, R, R_{c}\right)$ source code such that

$$
\begin{aligned}
\mathbb{E}\left[\rho_{n}\left(X^{n}, Y^{n}\right)\right] & \leq D+\varepsilon \\
Y^{n} & \sim \psi^{n} .
\end{aligned}
$$

In the rest of this paper $\psi$ will be kept fixed, so we drop referring to $\psi$ and simply write that $\left(R, R_{c}\right)$ is achievable. For $D \geq 0$ we let $\mathcal{R}(D)$ denote the set of all achievable $\left(R, R_{c}\right)$ pairs. The following theorem, which is the main result in this paper, characterizes the closure of this region in terms of an auxiliary random variable $U$ on alphabet $U$.

Theorem 1: For any $D \geq 0$ the closure $\operatorname{cl} \mathcal{R}(D)$ of $\mathcal{R}(D)$ is given by

$$
\begin{aligned}
\operatorname{cl} \mathcal{R}(D) & =\mathcal{L}(D) \\
& :=\left\{\begin{array}{c}
\left(R, R_{c}\right) \in \mathbb{R}^{2}: \exists P_{X, Y, U} \in \mathcal{M}(D) \\
\text { s.t. } R \geq I(X ; U), \\
R+R_{c} \geq I(Y ; U)
\end{array}\right\},
\end{aligned}
$$

where, for $\mathrm{X}=\mathrm{Y}$ finite,

$$
\mathcal{M}(D):=\left\{\begin{aligned}
P_{X, Y, U} & : P_{X}=\mu, P_{Y}=\psi \\
& \mathbb{E}[\rho(X, Y)] \leq D, X-U-Y, \\
& |\mathrm{U}| \leq|\mathrm{X}|+|\mathrm{Y}|+1
\end{aligned}\right\} .
$$

When $X=Y=\mathbb{R}$, the cardinality bound for $U$ in (4) is replaced by $U=\mathbb{R}$.

\section{A. Connections With Distributed Channel Synthesis}

As mentioned before, Cuff's work on distributed channel synthesis [17] is intrinsically related to our problem. The main objective of [17] is to simulate a memoryless channel by a system as in Fig. 1. To be more precise, let $Q(y \mid x)$ denote a given discrete memoryless channel with input alphabet $X$ and output alphabet $Y$ to be simulated (synthesized) for input $X$ having distribution $\mu$. Let $\pi=\mu Q$ be the joint distribution of the resulting input-output pair $(X, Y)$.

Definition 2 [17]: The pair $\left(R, R_{c}\right)$ is said to be achievable for synthesizing a memoryless channel $Q$ with input distribution $\mu$ if there exists a sequence of $\left(n, R, R_{c}\right)$ randomized source codes such that

$$
\lim _{n \rightarrow \infty}\left\|P_{X^{n}, Y^{n}}-\pi^{n}\right\|_{T V}=0,
$$

where $X^{n} \sim \mu^{n}$ is the memoryless source, $Y^{n}$ is the output of the decoder, $\pi^{n}$ is the $n$-fold product of $\pi=\mu Q=P_{X} Q$, and $\|\cdot\|_{T V}$ is the total variation distance for probability measures: $\|\gamma-v\|_{T V}:=\frac{1}{2} \sum_{v}|\gamma(v)-v(v)|$.

Theorem 2 [17, Th. II.1]: The closure $\mathcal{C}$ of the set of all achievable $\left(R, R_{C}\right)$ pairs is given by

$$
\mathcal{C}=\mathcal{S}:=\left\{\begin{aligned}
&\left(R, R_{C}\right) \in \mathbb{R}^{2}: \exists P_{X, Y, U} \in \mathcal{D} \\
& \text { s.t. } R \geq I(X ; U), \\
& R+R_{c} \geq I(X, Y ; U)
\end{aligned}\right\},
$$

where

$$
\mathcal{D}:=\left\{P_{X, Y, U}: P_{X, Y}=\pi, X-U-Y,|\mathrm{U}| \leq|\mathrm{X} \| \mathrm{Y}|+1\right\} .
$$

Moreover, the total variation error goes to zero exponentially fast with respect to $n$ in the interior of $\mathcal{C}$.

This result can be used to obtain an achievable rate region (inner bound) for our problem as follows: Let $\pi=P_{X, Y}$ be such that $P_{X}=\mu, P_{Y}=\psi$, and $\mathbb{E}[\rho(X, Y] \leq D$. Applying Theorem 2 with this input distribution and the channel induced by $P_{X, Y}$, consider an achievable rate pair $\left(R, R_{c}\right)$ in (6). Using basic results from optimal transport theory [19] one can show that (5) and the fact that $\mathbb{E}[\rho(X, Y)] \leq D$ imply the existence of a sequence of channels, to be used at the decoder side, that when fed with $Y^{n}$, produces output $\hat{Y}^{n}$ which has the exact distribution $\psi^{n}$ and which additionally satisfies

$$
\limsup _{n \rightarrow \infty} \mathbb{E}\left[\rho_{n}\left(X^{n}, \hat{Y}^{n}\right)\right] \leq D .
$$

Augmenting the channel synthesis code with these channels at the decoder side thus produces a sequence of valid codes for our problem, implying that the rate pair $\left(R, R_{c}\right)$ is achievable by our Definition 1.

Using the above argument, one can easily show that Cuff's result directly implies (without resorting to Theorem 1) the following inner bound for $\mathcal{R}(D)$. The proof is given in Appendix B.

Corollary 1: For any $D \geq 0$,

$$
\begin{aligned}
\operatorname{cl} \mathcal{R}(D) & \supset \mathcal{S}(D) \\
& :=\left\{\begin{array}{c}
\left(R, R_{C}\right) \in \mathbb{R}^{2}: \exists P_{X, Y, U} \in \mathcal{H}(D) \\
\text { s.t. } R \geq I(X ; U), \\
R+R_{c} \geq I(X, Y ; U)
\end{array}\right\},
\end{aligned}
$$


where

$$
\mathcal{H}(D):=\left\{\begin{aligned}
P_{X, Y, U}: & P_{X}=\mu, P_{Y}=\psi, \\
& \mathbb{E}[\rho(X, Y)] \leq D, X-U-Y, \\
& |\mathrm{U}| \leq|\mathrm{X} \| \mathrm{Y}|+1
\end{aligned}\right\} .
$$

In general, this inner bound is loose. For example, for $R_{c}=0$, only the constraint $R \geq I(X, Y ; U)$ is active in (8) since $I(X, Y ; U) \geq I(X ; U)$ always holds. Hence, letting $\mathcal{S}(D, 0)$ denote the set of $R \mathrm{~s}$ such that $(R, 0) \in \mathcal{S}(D)$, we obtain

$\mathcal{S}(D, 0)=\left\{R \in \mathbb{R}: \exists P_{X, Y, U} \in \mathcal{H}(D) \quad\right.$ s.t. $\left.R \geq I(X, Y ; U)\right\}$

The minimum of $\mathcal{S}(D, 0)$ can be written as

$$
\begin{aligned}
& \min \{R \in \mathcal{S}(D, 0)\} \\
& \quad=\min \left\{C(X ; Y): P_{X, Y} \in \mathcal{G}(D)\right\}=: C_{0}(\mu \| \psi, D),
\end{aligned}
$$

where $C(X ; Y)$ is Wyner's common information [20] defined for a given joint distribution $P_{X, Y}$ by

$$
C(X ; Y):=\inf _{U: X-U-Y} I(X, Y ; U),
$$

where the infimum is taken over all joint distributions $P_{X, Y, U}$ such that $U$ has a finite alphabet and $X-U-Y$. However, the resulting rate $C_{0}(\mu \| \psi, D)$ is not optimal as Example 1 in Section III-B will show.

The suboptimality of $C_{0}(\mu \| \psi, D)$ implies that a 'separated' solution which first finds an 'optimal' channel and then synthesizes this channel is not optimal for the constrained rate distortion problem we consider.

\section{Special CASES}

The extreme points at $R_{c}=\infty$ and $R_{c}=0$ of the rate region $\mathcal{L}(D)$ in our Theorem 1 are of particular interest. Let $\mathcal{L}\left(D, R_{c}\right)$ be the set of coding rates $R$ such that $\left(R, R_{c}\right) \in \mathcal{L}(D)$.

\section{A. Unlimited Common Randomness}

If $R_{c}=\infty$, then the effective constraint in (3) is $R \geq I(X ; U)$. This was the situation originally studied in [14] where it was assumed that the common randomness is of the form of a real-valued random variable that is uniformly distributed on the interval $[0,1]$. Since $I(X ; U) \geq I(X ; Y)$ by the data processing inequality and the condition $X-U-Y$, we can set $U=Y$ to obtain $\min \{R \in \mathcal{L}(D, \infty)\}=I(\mu \| \psi, D)$, recovering (1) and thus [14, Th. 7]. Furthermore, for the finite alphabet case whenever $R_{c} \geq H(Y \mid X)$, we have from (3) that $R+R_{c} \geq I(X ; U)+H(Y \mid X) \geq I(X ; Y)+H(Y \mid X)=H(Y) \geq$ $I(Y ; U)$, so the effective constraint is again $R \geq I(X ; U)$. Considering $(X, Y)$ such that $P_{X, Y}$ achieves the minimum in (1) and letting $U=Y$, we have

$$
\min \left\{R \in \mathcal{L}\left(D, R_{c}\right)\right\}=I(\mu \| \psi, D)
$$

or equivalently

$$
\mathcal{L}\left(D, R_{c}\right)=\mathcal{L}(D, \infty)
$$

Hence, $H(Y \mid X)$ is a sufficient common randomness rate above which the minimum communication rate does not decrease. In fact, letting

$$
R_{c}^{\min }=\min \left\{R_{c}: \mathcal{L}\left(D, R_{c}\right)=\mathcal{L}(D, \infty)\right\}
$$

we can determine $R_{c}^{\min }$ in terms of the so-called necessary conditional entropy [17], defined for a joint distribution $P_{X, Y}$ as

$$
H(Y \dagger X):=\min _{f: X-f(Y)-Y} H(f(Y) \mid X)
$$

where minimum is taken over all functions $f: \mathrm{Y} \rightarrow \mathrm{Y}$ such that $X-f(Y)-Y$. Using the discussion in [21, Sec. VII-C] one can verify that $R_{c}^{\min }$ is the minimum of $H(Y \dagger X)$ over all joint distributions of $(X, Y)$ achieving the minimum in (1). Indeed, for any joint distribution $P_{X, Y}$ achieving the minimum in (1), any function $f$ with the property

$$
f(y)=f(\tilde{y}) \Leftrightarrow P_{X \mid Y}(\cdot \mid y)=P_{X \mid Y}(\cdot \mid \tilde{y})
$$

minimizes $H(f(Y) \mid X)$ and satisfies $X-f(Y)-Y$; that is, $H(Y \dagger X)=H(f(Y) \mid X)$.

In general, for an arbitrary output distribution $\psi$, it may not be true that $H(Y \dagger X)=H(Y \mid X)$ for a joint distribution achieving the minimum in (1). Therefore, the Markov chain $X-Y-Y$ does not necessarily achieve $R_{c}^{\min }$. However, in the special case where the rate-distortion function

$$
R(D)=\min _{\psi} I(\mu \| \psi, D),
$$

is achieved by a unique output distribution $\psi$, we have the following proposition.

Proposition 1: Assume the rate-distortion function $R(D)$ is achieved by the unique output distribution $\psi$. Then $H(Y \dagger X)=H(Y \mid X)$ and the Markov chain $X-Y-Y$ (i.e., $U=Y$ ) achieves $R_{c}^{\min }$, where $(X, Y)$ achieve the rate-distortion function. In this case, $R+R_{c} \geq H(Y)$ when $R=R(D)$.

Proof: The proof is by contradiction. Suppose that $H(Y \dagger X)<H(Y \mid X)$. This implies the existence of a function $f$ with the property (13) and $H(f(Y) \mid X)<H(Y \mid X)$. In particular, there exist $\bar{y}, \tilde{y} \in \mathrm{Y}$ such that $\bar{y} \neq \tilde{y}$, $P_{Y}(\bar{y}), P_{Y}(\tilde{y})>0$, and $P_{X \mid Y}(\cdot \mid \bar{y})=P_{X \mid Y}(\cdot \mid \tilde{y})$. Without loss of generality we can assume $\mathbb{E}[\rho(X, Y) \mid Y=\bar{y}] \leq$ $\mathbb{E}[\rho(X, Y) \mid Y=\tilde{y}]$. Define a new pair $(\tilde{X}, \tilde{Y})$ with the joint distribution given by $P_{\tilde{X} \mid \tilde{Y}}=P_{X \mid Y}$ and $P_{\tilde{Y}}(y)=P_{Y}(y)$ if $y \in \mathrm{Y} \backslash\{\bar{y}, \tilde{y}\}$ and $P_{\tilde{Y}}(\bar{y})=P_{Y}(\bar{y})+P_{Y}(\tilde{y})\left(\operatorname{so}, P_{\tilde{Y}}(\tilde{y})=0\right)$. Hence, $\mathbb{E}[\rho(\tilde{X}, \tilde{Y})] \leq \mathbb{E}[\rho(X, Y)]$. Since $P_{X \mid Y}(\cdot \mid \bar{y})=$ $P_{X \mid Y}(\cdot \mid \tilde{y})$, we have $H(\tilde{X} \mid \tilde{Y})=H(X \mid Y)$ and $P_{\tilde{X}}=P_{X}=\mu$. Therefore, $I(\tilde{X} ; \tilde{Y})=I(X ; Y)$, and $(\tilde{X}, \tilde{Y})$ also achieves the rate distortion function. But, $P_{\tilde{Y}} \neq P_{Y}=\psi$, which is a contradiction.

\section{B. No Common Randomness}

Setting $R_{c}=0$ means that no common randomness is available. ${ }^{1}$ In this case (3) gives $R \geq \max (I(X ; U), I(Y ; U))$. Hence the minimum communication rate at distortion $D$ is given by

$$
\min \{R \in \mathcal{L}(D, 0)\}=I_{0}(\mu \| \psi, D),
$$

where

$$
\begin{aligned}
& I_{0}(\mu \| \psi, D) \\
& \quad:=\min \left\{\max (I(X ; U), I(Y ; U)): P_{X, Y, U} \in \mathcal{M}(D)\right\} .
\end{aligned}
$$

${ }^{1}$ Ram Zamir's question regarding the minimum coding rate in this special case has inspired our investigation of the general rate region $\mathcal{R}(D)$. 
Note that the minimum achievable coding rate $I_{0}(\mu \| \psi, D)$ is symmetric with respect to $\mu$ and $\psi$, i.e., $I_{0}(\mu \| \psi, D)=$ $I_{0}(\psi \| \mu, D)$. This is clear from the definition (14), but can also be deduced from the operational meaning of $I_{0}(\mu \| \psi, D)$ since in the absence of the common randomness $K$, the encoder-decoder structure is fully reversible. In general such symmetry no longer holds for $\min \left\{R \in \mathcal{R}\left(D, R_{c}\right)\right\}$ when $R_{c}>0$.

The following lemma states that $I_{0}(\mu \| \psi, D)$ is convex in $D$. The proof simply follows from a time-sharing argument and the operational meaning of $I_{0}(\mu \| \psi, D)$ implied by Theorem 1 . It is given in Appendix A.

Lemma 1: $I_{0}(\mu \| \psi, D)$ is a convex function of $D$.

An upper bound for $I_{0}(\mu \| \psi, D)$ can be given in terms of Wyner's common information. Since $\max (I(X ; U), I(Y ; U)) \leq I(X, Y ; U)$, we have $I_{0}(\mu \| \psi, D) \leq \min \left\{I(X, Y ; U): P_{X, Y, U} \in \mathcal{M}(D)\right\}$. The latter expression can also be written as

$$
\min \left\{C(X ; Y): P_{X, Y} \in \mathcal{G}(D)\right\}=: C_{0}(\mu \| \psi, D) .
$$

However, the resulting upper bound $I_{0}(\mu \| \psi, D) \leq$ $C_{0}(\mu \| \psi, D)$ is not tight in general as the next example shows.

Example 1: Let $\mathrm{X}=\mathrm{Y}=\{0,1\}$, and let $\mu=\psi=$ Bernoulli $(1 / 2)$, i.e., $\mu(0)=\mu(1)=\frac{1}{2}$. Assume the distortion measure $\rho$ is the Hamming distance $\rho(x, y)=1_{\{x \neq y\}}$ (which satisfies the assumptions in Section I-A). If $X \sim \mu$ and $Y \sim \psi$, then the channel $P_{Y \mid X}$ from $X$ to $Y$ must be Binary Symmetric Channel (BSC) with some crossover probability $a_{0}$, i.e.,

$$
P_{Y \mid X}(\cdot \mid 0)=1-P_{Y \mid X}(\cdot \mid 1)=\operatorname{Bernoulli}\left(a_{0}\right) .
$$

Wyner in [20, Sec. 3] showed that when $a_{0} \in[0,1 / 2]$,

$$
C(X ; Y)=1+h\left(a_{0}\right)-2 h\left(a_{1}\right),
$$

where $a_{1}=\frac{1}{2}\left(1-\sqrt{1-2 a_{0}}\right)$, and $h(\lambda)=-\lambda \log (\lambda)-(1-\lambda)$ $\log (1-\lambda)$. Define $C\left(a_{0}\right):=1+h\left(a_{0}\right)-2 h\left(a_{1}\right)$ which is decreasing and strictly concave in $[0,1 / 2]$. Notice that $\mathbb{E}[\rho(X, Y)]=a_{0}$ when $P_{Y \mid X}=\operatorname{BSC}\left(a_{0}\right)$. Hence, for any $D \in[0,1 / 2]$, we have

$$
\begin{aligned}
& C_{0}(\mu \| \psi, D) \\
& \quad=\min \left\{C(X ; Y): P_{X, Y} \in \mathcal{G}(D)\right\} \\
& \quad=\min \left\{C(X ; Y): P_{X}=\mu, P_{Y \mid X}=\operatorname{BSC}\left(a_{0}\right), a_{0} \leq D\right\} \\
& \quad=\min _{a_{0} \leq D} C\left(a_{0}\right)=C(D)
\end{aligned}
$$

implying that $C_{0}(\mu \| \psi, D)$ is strictly concave for $D \in[0,1 / 2]$. It is straightforward to prove that $C_{0}(\mu \| \psi, 0)=I_{0}(\mu \| \psi, 0)=1$ and $C_{0}(\mu \| \psi, 1 / 2)=I_{0}(\mu \| \psi, 1 / 2)=0$. Therefore, by Lemma 1 we have

$$
I_{0}(\mu \| \psi, D)<C_{0}(\mu \| \psi, D), \quad D \in(0,1 / 2) .
$$

\section{EXAMPLES}

In general determining the entire rate region $\mathcal{L}(D)$ in Theorem 1 seems to be difficult even for simple cases. In this section we obtain possibly suboptimal achievable rate regions (inner bounds) for two setups by restricting the channels $P_{U \mid X}$ and $P_{Y \mid U}$ so that the resulting optimization problem becomes manageable.

\section{A. Doubly Symmetric Binary Source}

In this section we obtain an inner bound for the setup in Example 1 (i.e., when $\mathrm{X}=\mathrm{Y}=\{0,1\}, \mu=\psi=$ Bernoulli(1/2), and $\rho$ the Hamming distance) by restricting the auxiliary random variable $U$ to be Bernoulli(1/2). Since $P_{X}=P_{U}=P_{Y}=\operatorname{Bernoulli}(1 / 2)$, for any $P_{X, Y, U} \in$ $\mathcal{M}(D)$, the channels $P_{U \mid X}$ and $P_{Y \mid U}$ must be $\operatorname{BSC}\left(a_{1}\right)$ and $\operatorname{BSC}\left(a_{2}\right)$, respectively, for some $a_{1}, a_{2} \in[0,1]$. Hence, since $\mathbb{E}[\rho(X, Y)]=a$ when $P_{X \mid Y}=\operatorname{BSC}(a)$, the resulting achievable rate region is

$$
\mathcal{L}_{S}(D)=\left\{\begin{array}{c}
\left(R, R_{c}\right) \in \mathbb{R}^{2}:\left(a_{1}, a_{2}\right) \in \Phi(D) \\
\text { s.t. } R \geq 1-h\left(a_{1}\right), \\
R+R_{c} \geq 1-h\left(a_{2}\right) .
\end{array}\right\},
$$

where

$$
\Phi(D):=\left\{\left(a_{1}, a_{2}\right) \in[0,1]^{2}: a_{1}+a_{2}-2 a_{1} a_{2} \leq D\right\} .
$$

Let us define $\varphi\left(a_{1}, a_{2}\right)=a_{1}+a_{2}-2 a_{1} a_{2}$. Note that since $\varphi\left(\frac{1}{2}+r, \frac{1}{2}+m\right)=\frac{1}{2}-2 r m$ and $h\left(\frac{1}{2}-r\right)=h\left(\frac{1}{2}+r\right)$ for any $r, m \in\left[\frac{-1}{2}, \frac{1}{2}\right]$; we may assume without loss of generality that $a_{1}, a_{2} \in\left[0, \frac{1}{2}\right]$ in the definition of $\Phi(D)$. Furthermore, since $\varphi\left(a_{1}, a_{2}\right)>D$ when $D<a_{1}<\frac{1}{2}$ or $D<a_{2}<\frac{1}{2}$, we can refine the definition of $\mathcal{L}_{S}(D)$ for $0 \leq D<\frac{1}{2}$ as

$$
\mathcal{L}_{s}(D)=\left\{\begin{array}{c}
\left(R, R_{c}\right) \in \mathbb{R}^{2}:\left(a_{1}, a_{2}\right) \in \Phi_{r}(D) \\
\text { s.t. } R \geq 1-h\left(a_{1}\right), \\
R+R_{c} \geq 1-h\left(a_{2}\right) .
\end{array}\right\},
$$

where

$$
\Phi_{r}(D):=\left\{\left(a_{1}, a_{2}\right) \in[0, D]^{2}: a_{1}+a_{2}-2 a_{1} a_{2} \leq D\right\} .
$$

Notice that for any fixed $a_{1},\left(a_{1}, a_{2}\right) \in \Phi_{r}(D)$ if and only if $a_{2} \leq \frac{D-a_{1}}{1-2 a_{1}}$, where the expression on the righthand side of the inequality is a concave function of $a_{1}$. Hence, $\Phi_{r}(D)$ is a convex region. In the remainder of this section we characterize the boundary $\bigcup_{R_{c}} \min \left\{R:\left(R, R_{c}\right) \in \mathcal{L}_{S}(D)\right\} \times\left\{R_{c}\right\}$ of $\mathcal{L}_{S}(D)$.

If $R_{c}=\infty$, then $(R, \infty) \in \mathcal{L}_{s}(D) \Leftrightarrow R \geq 1-h\left(a_{1}\right)$ where $a_{1} \in[0, D]$. Hence, the minimum $R$ is equal to $1-h(D)$ for $R_{c}=\infty$. Moreover, if $R=1-h(D)$ or equivalently $a_{1}=D$, then $\left(R, R_{c}\right) \in \mathcal{L}_{S}(D) \Leftrightarrow R_{c}+1-h(D) \geq 1-h\left(a_{2}\right)=$ $1-h(0)=1$ since $\left(D, a_{2}\right) \in \Phi_{r}(D)$ only if $a_{2}=0$. Hence, if and only if $R_{c} \geq h(D)$, then

$$
\min \left\{R:\left(R, R_{c}\right) \in \mathcal{L}_{s}(D)\right\}=1-h(D) .
$$

Note that since $1-h(D)$ is the rate-distortion function for the Bernoulli(1/2) source and $\psi=\operatorname{Bernoulli}(1 / 2)$ is the unique output distribution achieving this rate-distortion function, Proposition 1 implies that the inner bound we obtain in this section is tight for $R_{c} \geq h(D)=H(\psi)-(1-h(D))=R_{c}^{\min }$.

Recall that for an arbitrary $0 \leq R_{c}<h(D),\left(R, R_{c}\right) \in$ $\mathcal{L}_{S}(D) \Leftrightarrow R \geq \max \left\{1-h\left(a_{1}\right), 1-h\left(a_{2}\right)-R_{c}\right\}$ where $\left(a_{1}, a_{2}\right) \in$ $\Phi_{r}(D)$. We now prove that the minimum $R$ is attained when $1-h\left(a_{1}\right)=1-h\left(a_{2}\right)-R_{c}$ and $a_{1}+a_{2}-2 a_{1} a_{2}=D$. The second equality is clear since the binary entropy function $h$ is increasing in $[0, D]$. To prove the first claim by contradiction, let us assume (without loss of generality) that the minimum is achieved when $1-h\left(a_{1}\right)>1-h\left(a_{2}\right)-R_{c}$ (so $\min \{R$ : $\left.\left.\left(R, R_{c}\right) \in \mathcal{L}_{s}(D)\right\}=1-h\left(a_{1}\right)\right)$. Note that $\left(a_{1}, a_{2}\right) \in \Phi_{r}(D)$ if 


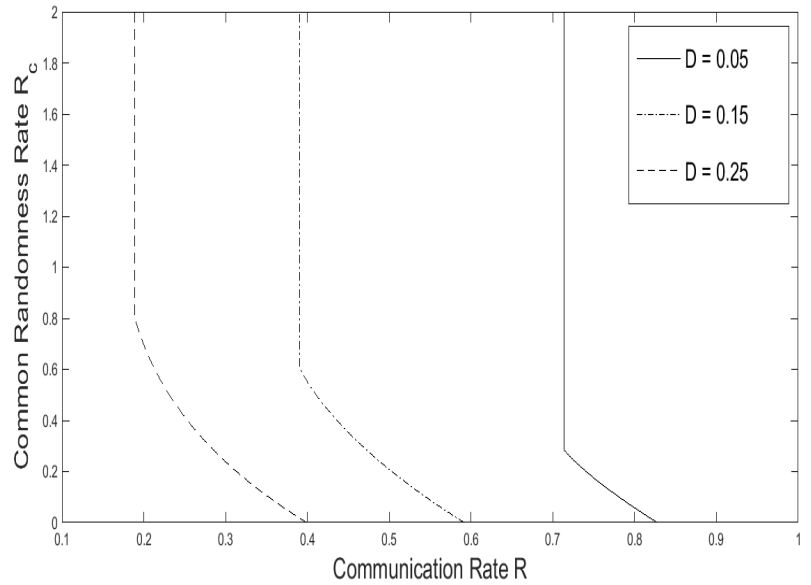

Fig. 2. $\mathcal{L}_{S}(D)$ for binary symmetric source at different distortion levels $D$. and only if $a_{2} \leq \frac{D-a_{1}}{1-2 a_{1}}$, where $\frac{D-a_{1}}{1-2 a_{1}}$ is a positive, decreasing, and concave function of $a_{1}$ in $(0, D)$. This and the fact that $h$ is increasing and continuous imply that there exist $\varepsilon_{1}, \varepsilon_{2}>0$ such that $\left(a_{1}+\varepsilon_{1}, a_{2}-\varepsilon_{2}\right) \in \Phi_{r}(D)$ and $1-h\left(a_{1}+\varepsilon_{1}\right) \geq$ $1-h\left(a_{2}-\varepsilon_{2}\right)-R_{c}$. But $\min \left\{R:\left(R, R_{c}\right) \in \mathcal{L}_{s}(D)\right\}=$ $1-h\left(a_{1}\right)>1-h\left(a_{1}+\varepsilon_{1}\right)$, which is a contradiction.

Hence, for all $D \in\left(0, \frac{1}{2}\right)$ the minimum coding rate when $0 \leq R_{c}<h(D)$ is given by

$$
\begin{aligned}
\min \left\{R:\left(R, R_{c}\right)\right. & \left.\in \mathcal{L}_{s}(D)\right\} \\
& =\min \left\{1-h\left(a_{1}\right):\left(a_{1}, a_{2}\right) \in \Pi\left(D, R_{c}\right)\right\}
\end{aligned}
$$

where

$$
\begin{aligned}
\Pi\left(D, R_{c}\right) & \\
& :=\left\{\begin{array}{r}
\left(a_{1}, a_{2}\right) \in \Phi_{r}(D): 1-h\left(a_{1}\right)=1-h\left(a_{2}\right)-R_{c} \\
\text { and } a_{1}+a_{2}-2 a_{1} a_{2}=D
\end{array}\right\} .
\end{aligned}
$$

Figure 2 shows the rate region $\mathcal{L}_{s}(D)$ for $D=0.25$, $D=0.15$, and $D=0.05$. At the boundary of $\mathcal{L}_{s}(D)$, the coding rate $R$ ranges from $1-h\left(a^{*}\right)=0.39,0.59,0.82$ bits $\left(a^{*}=\frac{1}{2}(1-\sqrt{1-2 D})\right)$ to $h(D)=0.19,0.4,0.72$ bits, respectively, while the common randomness rate $R_{c}$ ranges from 0 to $1-h(D)=0.81,0.6,0.28$ for $D=0.25, D=0.15$, and $D=0.05$, respectively.

\section{B. Gaussian Source}

Let $\mathrm{N}(m, \sigma)$ denote a Gaussian random variable with mean $m$ and variance $\sigma^{2}$ (similar notation will be used for the vector case). In this section, we obtain an inner bound for the case $\mathrm{X}=\mathrm{Y}=\mathbb{R}, \mu=\mathrm{N}\left(0, \sigma_{X}\right), \psi=\mathrm{N}\left(0, \sigma_{Y}\right)$, and $\rho$ is the squared error distortion (i.e., $\rho(x, y)=|x-y|^{2}$ ) by restricting $(X, U, Y)$ to be Gaussian (or, equivalently, restricting $(X, U)$ and $(U, Y)$ to be Gaussian since $X-U-Y)$.

Remark 1: Recall that for $R_{c}=\infty$, the minimum coding rate is given by (1). However if $X \sim \mathrm{N}\left(0, \sigma_{X}\right)$ and $Y \sim \mathrm{N}\left(0, \sigma_{Y}\right)$, then for any $P_{X, Y} \in \mathcal{G}(D)$, one has the lower bound

$$
\begin{aligned}
I(X ; Y) & =h(X)+h(Y)-h(X, Y) \\
& \geq \frac{1}{2} \log \left(2 \pi e \sigma_{X}^{2}\right)+\frac{1}{2} \log \left(2 \pi e \sigma_{Y}^{2}\right)-\log \left(2 \pi e \operatorname{det}(C)^{\frac{1}{2}}\right),
\end{aligned}
$$

where $C$ is the covariance matrix of $(X, Y)$. The equality is achieved when $(X, Y)$ is jointly Gaussian [22, Th. 8.6.5].
Hence, we can restrict $(X, Y)$ to be Gaussian in the definition of $I(\mu \| \psi, D)$, i.e.,

$$
I(\mu \| \psi, D):=\min \left\{I(X, Y): P_{X, Y} \in \mathcal{G}_{g}(D)\right\},
$$

where

$$
\mathcal{G}_{g}(D):=\left\{P_{X, Y} \in \mathcal{G}(D): P_{X, Y}=\mathrm{N}(0, C) \text { for some } C\right\} .
$$

This implies that the inner bound we obtain in this section is tight for $R_{c}=\infty$ (i.e., $\left.\mathcal{L}_{s}(D, \infty)=\mathcal{L}(D, \infty)\right)$. $\mathcal{L}(D, \infty)$ for the case $\mu=\psi=\mathrm{N}(0, \sigma)$ was derived in [11, Proposition 2].

Note that without loss of generality we can take $U$ to have zero mean and unit variance. Indeed, let $\tilde{U}=\left(U-\delta_{U}\right) / \sigma_{U}$. Then $\tilde{U} \sim \mathrm{N}(0,1), X-\tilde{U}-Y$, and $(X, \tilde{U}, Y)$ is Gaussian with $I(X ; U)=I(X ; \tilde{U})$ and $I(Y ; U)=I(Y ; \tilde{U})$. Hence, in the remainder of this section, we assume $U \sim \mathrm{N}(0,1)$.

Let us write $U=a X+V$ and $Y=b U+W$, where $a, b \in \mathbb{R}$, and $V \sim \mathrm{N}\left(0, \sigma_{V}\right), W \sim \mathrm{N}\left(0, \sigma_{W}\right)$, and $(X, V, W)$ are independent. With this representation, the constraints in the definition of the achievable rate region become

$$
\begin{aligned}
1 & =a^{2} \sigma_{X}^{2}+\sigma_{V}^{2}, \\
\sigma_{Y}^{2} & =b^{2}+\sigma_{W}^{2}, \\
(1-a b)^{2} \sigma_{X}^{2}+b^{2} \sigma_{V}^{2}+\sigma_{W}^{2} & \leq D,
\end{aligned}
$$

Then, if we substitute $\sigma_{V}^{2}=1-a^{2} \sigma_{X}^{2} \geq 0$ and $\sigma_{W}^{2}=\sigma_{Y}^{2}-b^{2} \geq 0$ into the last equation, we can write the distortion constraint as

$$
\sigma_{X}^{2}+\sigma_{Y}^{2}-2 a b \sigma_{X}^{2} \leq D
$$

Since

$$
\begin{aligned}
I(X ; U) & =H(X)+H(U)-H(X, U) \\
& =\frac{1}{2} \log \left(2 \pi \mathrm{e} \sigma_{X}^{2}\right)+\frac{1}{2} \log (2 \pi \mathrm{e})-\log \left(2 \pi \mathrm{e} \operatorname{det}\left(C_{X}\right)^{\frac{1}{2}}\right) \\
& =\frac{1}{2} \log \left(\frac{1}{\left(1-a^{2} \sigma_{X}^{2}\right)}\right)
\end{aligned}
$$

and

$$
\begin{aligned}
I(Y ; U) & =H(Y)+H(U)-H(Y, U) \\
& =\frac{1}{2} \log \left(2 \pi \mathrm{e} \sigma_{Y}^{2}\right)+\frac{1}{2} \log (2 \pi \mathrm{e})-\log \left(2 \pi \mathrm{e} \operatorname{det}\left(C_{Y}\right)^{\frac{1}{2}}\right) \\
& =\frac{1}{2} \log \left(\frac{\sigma_{Y}^{2}}{\left(\sigma_{Y}^{2}-b^{2}\right)}\right),
\end{aligned}
$$

where $C_{X}$ is the covariance matrix of $(X, U)$ and $C_{Y}$ is the covariance matrix of $(Y, U)$, the resulting achievable rate region can be written as

$$
\mathcal{L}_{s}(D)=\left\{\begin{aligned}
\left(R, R_{c}\right) \in \mathbb{R}^{2}:(a, b) \in \Psi(D) \\
\text { s.t. } R \geq \frac{1}{2} \log \left(\frac{1}{\left(1-a^{2} \sigma_{X}^{2}\right)}\right), \\
R+R_{c} \geq \frac{1}{2} \log \left(\frac{\sigma_{Y}^{2}}{\left(\sigma_{Y}^{2}-b^{2}\right)}\right) .
\end{aligned}\right\},
$$

where

$\Psi(D)$

$$
:=\left\{(a, b) \in\left[0, \sigma_{X}^{-1}\right] \times\left[0, \sigma_{Y}\right]: \sigma_{X}^{2}+\sigma_{Y}^{2}-2 a b \sigma_{X}^{2} \leq D\right\} .
$$

Note that the region $\Psi(D)$ is convex. Let us define $I_{1}(a)=\log \left(\frac{1}{\left(1-a^{2} \sigma_{X}^{2}\right)}\right)$ and $I_{2}(b)=\log \left(\frac{\sigma_{Y}^{2}}{\left(\sigma_{Y}^{2}-b^{2}\right)}\right)$; then 


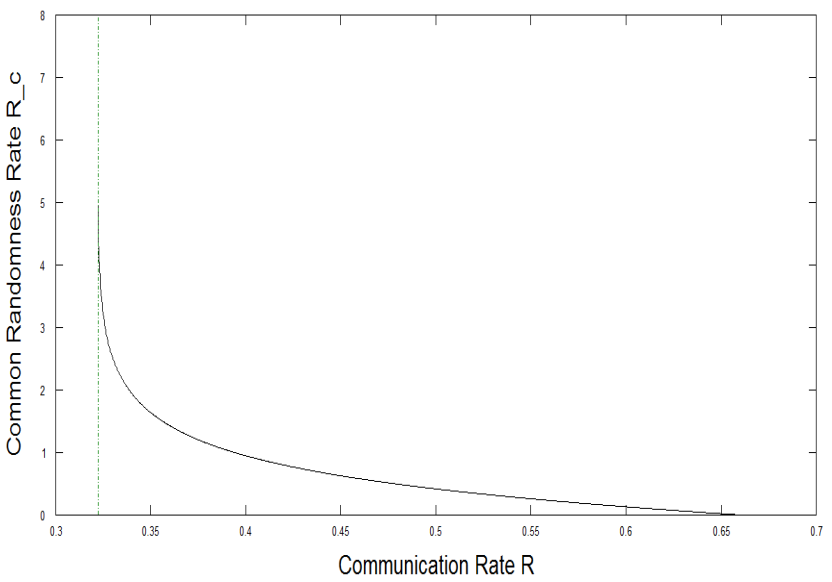

Fig. 3. $\mathcal{L}_{S}(D)$ for Gaussian source for $D=0.8$.

$I_{1}$ and $I_{2}$ are increasing functions. As in Section IV-A, we characterize the boundary $\bigcup_{R_{c}} \min \left\{R:\left(R, R_{c}\right) \in \mathcal{L}_{s}(D)\right\} \times$ $\left\{R_{c}\right\}$ of $\mathcal{L}_{s}(D)$.

If $R_{c}=\infty$, then $(R, \infty) \in \mathcal{L}_{S}(D) \Leftrightarrow R \geq I_{1}(a)$ where $(a, b) \in\left[0, \sigma_{X}^{-1}\right] \times\left[0, \sigma_{Y}\right]$ and $\sigma_{X}^{2}+\sigma_{Y}^{2}-2 a b \sigma_{X}^{2} \leq D$. Using the monotonicity of $I_{1}$ and the distortion constraint, it is straightforward to show that

$$
\min \left\{R:(R, \infty) \in \mathcal{L}_{s}(D)\right\}=I_{1}\left(\frac{\sigma_{X}^{2}+\sigma_{Y}^{2}-D}{2 \sigma_{X}^{2} \sigma_{Y}}\right) .
$$

By Remark 1, this is the minimum coding rate (i.e., rate-distortion function) for $R_{c}=\infty$.

When $0 \leq R_{c}<\infty$ is arbitrary, we can use the same technique as in Section IV-A to prove that the minimum of $R$ is attained when $I_{1}(a)=I_{2}(b)-R_{c}$ and $\sigma_{X}^{2}+\sigma_{Y}^{2}-2 a b \sigma_{X}^{2}=D$ ( $I_{1}$ and $I_{2}$ are increasing continuous functions and $\Psi(D)$ is a convex region with nonempty interior in the upper-right corner of the rectangle $\left.\left[0, \sigma_{X}^{-1}\right] \times\left[0, \sigma_{Y}\right]\right)$. As a consequence, we can describe the minimum coding rate when $0 \leq R_{c}<\infty$ as follows:

$\min \left\{R:\left(R, R_{c}\right) \in \mathcal{L}_{s}(D)\right\}=\min \left\{I_{1}(a):(a, b) \in \Lambda\left(D, R_{c}\right)\right\}$

where

$\Lambda\left(D, R_{c}\right):=\left\{(a, b) \in \Psi(D): \begin{array}{ll}I_{1}(a)=I_{2}(b)-R_{c} \text { and } \\ & \sigma_{X}^{2}+\sigma_{Y}^{2}-2 a b \sigma_{X}^{2}=D\end{array}\right\}$.

Figure 3 shows the rate region $\mathcal{L}_{S}(D)$ for $\sigma_{X}=\sigma_{Y}=1$ and $D=0.8$. At the boundary of $\mathcal{L}_{S}(D)$, the coding rate $R$ ranges from $I_{1}\left(\sqrt{\frac{2-D}{2}}\right)=0.65$ bits to $I_{1}\left(\frac{2-D}{2}\right)=0.32$ bits while the common randomness rate $R_{c}$ ranges from 0 to infinity.

\section{TWO VARIATIONS}

In this section we consider two variations of the ratedistortion problem defined in Section II. Throughout this section we assume that the source alphabet $X$ and the reproduction alphabet $Y$ are finite.

\section{A. Rate Region With Empirical Distribution Constraint}

First, we investigate the effect on the achievable rate region of relaxing the strict output distribution constraint on $Y^{n}$ and requiring only that the empirical output distribution $p_{Y^{n}}$ converges to the distribution $\psi$.

Definition 3: For any positive real number $D$ and desired output distribution $\psi$, the pair $\left(R, R_{c}\right)$ is said to be empirically achievable if there exists a sequence of $\left(n, R, R_{c}\right)$ randomized source codes such that

$$
\begin{aligned}
\limsup _{n \rightarrow \infty} & \mathbb{E}\left[\rho_{n}\left(X^{n}, Y^{n}\right)\right] \leq D, \\
\left\|p_{Y^{n}}-\psi\right\|_{T V} & \rightarrow 0 \text { in probability as } n \rightarrow \infty .
\end{aligned}
$$

For any $D \geq 0$ we let $\mathcal{R}_{e}(D)$ denote the set of all empirically achievable rate pairs $\left(R, R_{c}\right)$, and define $\mathcal{R}_{e}\left(D, R_{c}\right)$ as the set of coding rates $R$ such that $\left(R, R_{c}\right) \in \mathcal{R}_{e}(D)$.

This setup is motivated by the work of Cuff et al. [21, Sec. II] on empirical coordination. The main objective of $[21$, Sec. II] is to empirically simulate a memoryless channel by a system as in Fig. 1. To be more precise, let $Q(y \mid x)$ denote a given discrete memoryless channel with input alphabet $X$ and output alphabet $Y$ to be simulated (synthesized) for input $X$ having distribution $\mu$. Let $\pi=\mu Q$ be the joint distribution of the resulting input-output pair $(X, Y)$.

Definition 4: The pair $\left(R, R_{c}\right)$ is said to be achievable for empirically synthesizing a memoryless channel $Q$ with input distribution $\mu$ if there exists a sequence of $\left(n, R, R_{c}\right)$ randomized source codes such that

$$
\lim _{n \rightarrow \infty}\left\|p_{X^{n}, Y^{n}}-\pi\right\|_{T V}=0 \text { in probability. }
$$

Let $\mathcal{C}_{e}$ denote the the set of all achievable $\left(R, R_{C}\right)$ pairs and let $\mathcal{C}_{e}\left(R_{c}\right)$ denote the set of all rates $R$ such that $\left(R, R_{c}\right) \in \mathcal{C}_{e}$. The following theorem, which is a combination of [21, Th. 2 and 3], characterizes the entire set $\mathcal{C}_{e}$.

Theorem 3: The set $\mathcal{C}_{e}$ of all achievable $\left(R, R_{c}\right)$ is given by

$$
\mathcal{C}_{e}=\left\{\begin{aligned}
\left(R, R_{C}\right) \in \mathbb{R}^{2}: \exists P_{X, Y} \in \mathcal{G} \\
\text { s.t. } R \geq I(X ; Y)
\end{aligned}\right\},
$$

where

$$
\mathcal{G}:=\left\{P_{X, Y}: P_{X, Y}=\pi\right\} .
$$

Hence, $\mathcal{C}_{e}\left(R_{c}\right)=\mathcal{C}_{e}(0)$ for any $R_{c}$.

Using the above theorem and the arguments in [21, Sec. VII], one can show that the set of empirically achievable rate pairs $\left(R, R_{C}\right)$ at the distortion level $D$ can be described as:

Theorem 4: For any $D \geq 0$ we have

$$
\begin{aligned}
\mathcal{R}_{e}(D, 0) & =\mathcal{L}(D, \infty), \\
\mathcal{R}_{e}\left(D, R_{c}\right) & =\mathcal{R}_{e}(D, 0) \text { for all } R_{c} .
\end{aligned}
$$

In other words, $\mathcal{R}_{e}(D)=\mathcal{L}(D, \infty) \times[0, \infty)$.

The proof of Theorem 4 is given in Appendix C. Note that (17) states that unlike in the original problem defined in Section II, here common randomness cannot decrease the necessary coding rate.

\section{B. Deterministic-Decoder Rate Region}

In this section we investigate the effect on the rate region of private randomness used by the decoder. Namely, we determine the achievable rate region for a randomized source 
code having no (private) randomness at the decoder, i.e., when the decoder $F$ is a deterministic function of random variables $J$ and $K$. We call such a code a randomized source code with deterministic decoder. In this setup, since the encoder can reconstruct the output $Y^{n}$ of the decoder by reading off $J$ and $K$, the common randomness $K$ may be interpreted as feedback from the output of the decoder to the encoder [23, page 5].

Definition 5: For any positive real number $D$ and desired output distribution $\psi$, the pair $\left(R, R_{c}\right)$ is said to be achievable with a deterministic decoder if there exists a sequence of $\left(n, R, R_{c}\right)$ randomized source codes with a deterministic decoder such that

$$
\begin{aligned}
& \limsup _{n \rightarrow \infty} \mathbb{E}\left[\rho_{n}\left(X^{n}, Y^{n}\right)\right] \leq D, \\
& \lim _{n \rightarrow \infty}\left\|P_{Y^{n}}-\psi^{n}\right\|_{T V}=0 .
\end{aligned}
$$

Note that here we relax the strict i.i.d. output distribution constraint, because without private randomness at the decoder, some output distributions cannot be exactly achieved for finite rates $\left(R, R_{c}\right)$. Indeed, this is the case when the probabilities of the output distribution are irrational and the input distribution has rational probabilities.

For any $D \geq 0$ we let $\mathcal{R}_{d d}(D)$ denote the set of all achievable $\left(R, R_{c}\right)$ pairs with deterministic decoder. The following theorem, proved in Appendix D, characterizes the closure of this set.

Theorem 5: For any $D \geq 0$,

$$
\operatorname{cl} \mathcal{R}_{d d}(D)=\left\{\begin{aligned}
&\left(R, R_{c}\right) \in \mathbb{R}^{2}: \exists P_{X, Y} \in \mathcal{G}(D) \\
& \text { s.t. } R \geq I(X ; Y), \\
& R+R_{c} \geq H(Y)
\end{aligned}\right\} .
$$

Remark 2:

(a) Note that the rate region in Theorem 5 can equivalently be given by

$$
\begin{aligned}
& \operatorname{cl} \mathcal{R}_{d d}(D) \\
& \quad=\left\{\begin{aligned}
\left(R, R_{c}\right) \in \mathbb{R}^{2}: \exists P_{X, Y, U} \in \mathcal{M}(D) \\
\text { s.t. } R \geq I(X ; U), \\
R+R_{c} \geq H(Y)
\end{aligned}\right\} .
\end{aligned}
$$

Therefore, $\mathcal{L}(D) \supset \mathrm{cl} \mathcal{R}_{d d}(D)$.

(b) It is important to note that if we allow the decoder to use private randomness while preserving the output distribution constraint (18), one can prove that the resulting achievable rate region is $\mathcal{L}(D)$. In this case, the only part to prove is the converse, since the achievability is obvious. However, the converse can be proven by using a similar technique as in $[17$, Sec. VI]. Hence, if we allow the decoder to use private randomness, replacing the strict output distribution constraint in the Definition 1 with (18) does not change the achievable rate region.

(c) Since $\mathcal{L}(D) \supset \mathrm{cl} \mathcal{R}_{d d}(D)$, where the inclusion is strict in general, private randomness can indeed replace a part of the common randomness to decrease the necessary coding rate when the common randomness rate is less than $R_{c}^{\mathrm{min}}$.

\section{Proof of Theorem 1}

Our proof relies on techniques developed by Cuff in [17]. In particular, in the achievability part, we apply the 'likelihood encoder' of [17] and [21] which is an elegant alternative to the standard random coding argument. The converse part of the proof is an appropriately modified version of the converse argument in [17]; however, in our setup this technique also works in the continuous alphabet case, while in [17] the finite alphabet assumption seem quite difficult to relax.

\section{A. Achievability for Discrete Alphabets}

Assume that $\left(R, R_{C}\right)$ is in the interior of $\mathcal{L}(D)$. Then there exists $P_{X, Y, U} \in \mathcal{M}(D)$ such that $R>I(X ; U)$ and $R+R_{c}>$ $I(Y ; U)$. The method used in this part of the proof comes from $[17$, Sec. V] where instead of explicitly constructing the encoder-decoder pair, a joint distribution was constructed from which the desired encoder-decoder behavior is established.

In this section, distributions which depend on realizations of some random variable (e.g., random codebook) will be denoted as bold upper case letters, but without referring to the corresponding realization for notational simplicity.

For each $n$, generate a random 'codebook' $\mathcal{C}_{n}:=\left\{U^{n}(j, k)\right\}$ of $u^{n}$ sequences independently drawn from $P_{U}^{n}$ and indexed by $(j, k) \in\left[2^{n R}\right] \times\left[2^{n R_{c}}\right]$. For each realization $\left\{u^{n}(j, k)\right\}$ of $\mathcal{C}_{n}$, define a distribution $\boldsymbol{\Gamma}_{X^{n}, Y^{n}, J, K}$ such that $(J, K)$ is uniformly distributed on $\left[2^{n R}\right] \times\left[2^{n R_{c}}\right]$ and $\left(X^{n}, Y^{n}\right)$ is the output of the stationary and memoryless channel $P_{X, Y \mid U}^{n}$ when we feed it with $u^{n}(J, K)$, i.e.,

$\boldsymbol{\Gamma}_{X^{n}, Y^{n}, J, K}\left(x^{n}, y^{n}, j, k\right):=\frac{1}{2^{n\left(R+R_{c}\right)}} P_{X, Y \mid U}^{n}\left(x^{n}, y^{n} \mid u^{n}(j, k)\right)$.

Here, $\left\{\boldsymbol{\Gamma}_{X^{n}, Y^{n}, J, K}\right\}_{n \geq 1}$ are the distributions from which we derive a sequence of encoder-decoder pairs which for all $n$ large enough almost meet the requirements in Definition 1.

Lemma 2 (Soft Covering Lemma [17, Lemma IV.1]): Let $P_{V, W}=P_{V} P_{W \mid V}$ be the joint distribution of some random vector $(V, W)$ on $\mathrm{V} \times \mathrm{W}$, where $P_{V}$ is the marginal on $\mathrm{V}$ and $P_{W \mid V}$ is the conditional probability on $\mathrm{W}$ given $\mathrm{V}$. For each $n$, generate the set $\mathcal{B}_{n}=\left\{V^{n}(i)\right\}$ of $v^{n}$ sequences independently drawn from $P_{V}^{n}$ and indexed by $i \in\left[2^{n R}\right]$. Let us define a random measure on $\mathrm{W}^{n}$ as

$$
\mathbf{P}_{W^{n}}\left(w^{n}\right):=\frac{1}{\left|\mathcal{B}_{n}\right|} \sum_{i=1}^{\left|\mathcal{B}_{n}\right|} P_{W^{n} \mid V^{n}}\left(w^{n} \mid V^{n}(i)\right),
$$

where $P_{W^{n} \mid V^{n}}=\prod_{i=1}^{n} P_{W \mid V}$. If $R \geq I(V ; W)$, then we have

$$
\mathbb{E}_{\mathcal{B}_{n}}\left[\left\|\mathbf{P}_{W^{n}}-P_{W}^{n}\right\|_{T V}\right] \leq \frac{3}{2} \exp \{-\kappa n\},
$$

for some $\kappa>0$.

Since $R+R_{c}>I(Y ; U)$, by the soft covering lemma

$$
\mathbb{E}_{\mathcal{C}_{n}}\left[\left\|\boldsymbol{\Gamma}_{Y^{n}}-P_{Y}^{n}\right\|_{T V}\right] \leq \frac{3}{2} \exp \{-c n\},
$$

where $c>0$ and $\mathbb{E}_{\mathcal{C}_{n}}$ denotes expectation with respect to the distribution of $\mathcal{C}_{n}$. Note that for any fixed $k$, the collection 
$\mathcal{C}_{n}(k):=\left\{U^{n}(j, k)\right\}_{j}$ is a random codebook of size $2^{n R}$. Since $R>I(X ; U)$, the soft covering lemma again gives

$$
\mathbb{E}_{\mathcal{C}_{n}(k)}\left[\left\|\boldsymbol{\Gamma}_{X^{n} \mid K=k}-P_{X}^{n}\right\|_{T V}\right] \leq \frac{3}{2} \exp \{-d n\},
$$

where $d>0$ (same for all $k$ ) and $\mathbb{E}_{\mathcal{C}_{n}(k)}$ denotes expectation with respect to the distribution of $\mathcal{C}_{n}(k)$. Then, by the definition of total variation, we have

$$
\begin{aligned}
\mathbb{E}_{\mathcal{C}_{n}}\left[\left\|\boldsymbol{\Gamma}_{X^{n}, K}-\frac{1}{2^{n R_{c}}} P_{X}^{n}\right\|_{T V}\right] \\
:=\mathbb{E}_{\mathcal{C}_{n}}\left[\frac{1}{2} \sum_{x^{n}, k}\left|\boldsymbol{\Gamma}_{X^{n}, K}\left(x^{n}, k\right)-\frac{1}{2^{n R_{c}}} P_{X}^{n}\left(x^{n}\right)\right|\right] \\
=\frac{1}{2^{n R_{c}}} \mathbb{E}_{\mathcal{C}_{n}}\left[\frac{1}{2} \sum_{x^{n}, k}\left|\boldsymbol{\Gamma}_{X^{n} \mid K}\left(x^{n} \mid k\right)-P_{X}^{n}\left(x^{n}\right)\right|\right] \\
=\frac{1}{2^{n R_{c}}} \sum_{k} \mathbb{E}_{\mathcal{C}_{n}(k)}\left[\left\|\boldsymbol{\Gamma}_{X^{n} \mid K=k}-P_{X}^{n}\right\|_{T V}\right] \\
\leq \frac{3}{2} \exp \{-d n\} .
\end{aligned}
$$

Furthermore, the expected value (taken with respect to the distribution of $\mathcal{C}_{n}$ ) of the distortion induced by $\boldsymbol{\Gamma}_{X^{n}, Y^{n}}$ is upper bounded by $D$ as a result of the symmetry in the construction of $\mathcal{C}_{n}$, i.e.,

$$
\begin{aligned}
\mathbb{E}_{\mathcal{C}_{n}} & {\left[\sum_{x^{n}, y^{n}} \rho_{n}\left(x^{n}, y^{n}\right) \boldsymbol{\Gamma}_{X^{n}, Y^{n}}\left(x^{n}, y^{n}\right)\right] } \\
= & \mathbb{E}_{\mathcal{C}_{n}}\left[\sum_{j, k} \sum_{x^{n}, y^{n}} \rho_{n}\left(x^{n}, y^{n}\right) \boldsymbol{\Gamma}_{X^{n}, Y^{n}, J, K}\left(x^{n}, y^{n}, j, k\right)\right] \\
= & \sum_{x^{n}, y^{n}} \rho_{n}\left(x^{n}, y^{n}\right) \sum_{j, k} \mathbb{E}_{\mathcal{C}_{n}}\left[\boldsymbol{\Gamma}_{X^{n}, Y^{n}, J, K}\left(x^{n}, y^{n}, j, k\right)\right] \\
= & \sum_{x^{n}, y^{n}} \rho_{n}\left(x^{n}, y^{n}\right) P_{X, Y}^{n}\left(x^{n}, y^{n}\right) \leq D,
\end{aligned}
$$

where the last equality follows from the symmetry and the independence in the codebook construction, and the last inequality follows from the definition of $\mathcal{M}(D)$.

Now, since $\boldsymbol{\Gamma}_{Y^{n}, J \mid X^{n}, K}=\boldsymbol{\Gamma}_{J \mid X^{n}, K} \boldsymbol{\Gamma}_{Y^{n} \mid J, K}$, we define a randomized $\left(n, R, R_{c}\right)$ source code such that it has the encoder-decoder pair $\left(\boldsymbol{\Gamma}_{J \mid X^{n}, K}, \boldsymbol{\Gamma}_{Y^{n} \mid J, K}\right)$. Hence, $\left(n, R, R_{c}\right)$ depends on the realization of $\mathcal{C}_{n}$. Let $\mathbf{P}_{X^{n}, Y^{n}, J, K}$ denote the distribution induced by $\left(n, R, R_{c}\right)$, i.e.,

$$
\begin{aligned}
& \mathbf{P}_{X^{n}, Y^{n}, J, K}\left(x^{n}, y^{n}, j, k\right) \\
& \quad:=\frac{1}{2^{n R_{c}}} P_{X}^{n}\left(x^{n}\right) \boldsymbol{\Gamma}_{Y^{n}, J \mid X^{n}, K}\left(y^{n}, j \mid x^{n}, k\right) .
\end{aligned}
$$

If two distributions are passed through the same channel, then the total variation between the joint distributions is the same as the total variation between the input distributions [17, Lemma V.2]. Hence, by (24)

$$
\mathbb{E}_{\mathcal{C}_{n}}\left[\left\|\boldsymbol{\Gamma}_{X^{n}, Y^{n}, K, J}-\mathbf{P}_{X^{n}, Y^{n}, K, J}\right\|_{T V}\right] \leq \frac{3}{2} \exp \{-d n\} .
$$

Then, (25) and (26) give

$$
\mathbb{E}_{\mathcal{C}_{n}}\left[\sum_{x^{n}, y^{n}} \rho_{n}\left(x^{n}, y^{n}\right) \mathbf{P}_{X^{n}, Y^{n}}\left(x^{n}, y^{n}\right)\right] \leq D+\alpha \exp \{-d n\},
$$

where $\alpha=\rho_{\max } \frac{3}{2}$. By virtue of the properties of total variation distance, (22) and (26) also imply

$$
\begin{aligned}
\mathbb{E}_{\mathcal{C}_{n}}\left[\left\|\mathbf{P}_{Y^{n}}-P_{Y}^{n}\right\|_{T V}\right] \\
\quad \leq \mathbb{E}_{\mathcal{C}_{n}}\left[\left\|\mathbf{P}_{Y^{n}}-\boldsymbol{\Gamma}_{Y^{n}}\right\|_{T V}\right]+\mathbb{E}_{\mathcal{C}_{n}}\left[\left\|\boldsymbol{\Gamma}_{Y^{n}}-P_{Y}^{n}\right\|_{T V}\right] \\
\quad \leq \frac{3}{2} \exp \{-d n\}+\frac{3}{2} \exp \{-c n\} \\
\quad=\alpha_{n} \exp \{-d n\},
\end{aligned}
$$

where (without any loss of generality) we assumed $d<c$ and where $\alpha_{n}:=\frac{3}{2}(1+\exp \{-(c-d) n\}) \leq 2$ if $n$ is large enough.

Define the following functions of the random codebook $\mathcal{C}_{n}$ :

$$
\begin{aligned}
D\left(\mathcal{C}_{n}\right) & :=\sum_{x^{n}, y^{n}} \rho_{n}\left(x^{n}, y^{n}\right) \mathbf{P}_{X^{n}, Y^{n}}\left(x^{n}, y^{n}\right), \\
G\left(\mathcal{C}_{n}\right) & :=\left\|\mathbf{P}_{Y^{n}}-P_{Y}^{n}\right\| .
\end{aligned}
$$

Thus, the expectations of $D\left(\mathcal{C}_{n}\right)$ and $G\left(\mathcal{C}_{n}\right)$ satisfy (27) and (28), respectively. For any $\delta \in(0, d)$, Markov's inequality gives

$$
\begin{aligned}
\operatorname{Pr}\left\{G\left(\mathcal{C}_{n}\right) \leq \exp \{-\delta n\}\right\} & \geq 1-\frac{\alpha_{n} \exp \{-d n\}}{\exp \{-\delta n\}}, \\
\operatorname{Pr}\left\{D\left(\mathcal{C}_{n}\right) \leq D+\delta\right\} & \geq 1-\frac{D+\alpha \exp \{-d n\}}{D+\delta} .
\end{aligned}
$$

Since

$$
\begin{array}{r}
\lim _{n \rightarrow \infty}\left(2-\frac{\alpha_{n} \exp \{-d n\}}{\exp \{-\delta n\}}-\frac{D+\beta \exp \{-d n\}}{D+\delta}\right) \\
=2-\frac{D}{D+\delta}>1,
\end{array}
$$

there exists a positive $N(\delta)$ such that for $n \geq N(\delta)$, we have

$$
\operatorname{Pr}\left\{\left(D\left(\mathcal{C}_{n}\right) \leq D+\delta\right) \bigcap\left(G\left(\mathcal{C}_{n}\right) \leq \exp \{-\delta n\}\right)\right\}>0 .
$$

This means that for each $n \geq N(\delta)$, there is a realization of $\mathcal{C}_{n}$ which gives

$$
\begin{aligned}
\sum_{x^{n}, y^{n}} \rho_{n}\left(x^{n}, y^{n}\right) \mathbf{P}_{X^{n}, Y^{n}}\left(x^{n}, y^{n}\right) & \leq D+\delta \\
\left\|\mathbf{P}_{Y^{n}}-P_{Y}^{n}\right\| & \leq \exp \{-\delta n\} .
\end{aligned}
$$

Hence, the sequence of $\left(n, R, R_{c}\right)$ randomized source codes corresponding to these realizations almost satisfies the achievability constraints. Next we can slightly modify this coding scheme so that the code exactly satisfies the i.i.d. output distribution constraint $Y^{n}=\psi^{n}=P_{Y}^{n}$ while having distortion upper bounded by $D+\delta$.

Before presenting this modification, we pause to define the notion of optimal coupling and the optimal transportation cost as they will play an important role in the sequel. Let $\pi, \lambda$ be probability measures over finite or continuous alphabets $W$ and $\mathrm{V}$, respectively. The optimal transportation cost $\hat{T}(\pi, \lambda)$ between $\pi$ and $\lambda$ (see [19]) with respect to a cost function $c: \mathrm{V} \times \mathrm{W} \rightarrow[0, \infty)$ is defined by

$$
\hat{T}(\pi, \lambda)=\inf \{\mathbb{E}[c(V, W)]: V \sim \pi, W \sim \lambda\},
$$

where the infimum is taken over all joint distribution of pairs of random variables $(V, W)$ satisfying the given marginal distribution constraints. The distribution achieving $\hat{T}(\pi, \lambda)$ is 


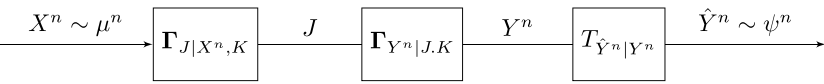

Fig. 4. Randomized source code used in the achievability proof for discrete alphabets.

called an optimal coupling of $\pi$ and $\lambda$. Somewhat informally, we also call the corresponding conditional probability on $\mathrm{W}$ given $\mathrm{V}$ an optimal coupling. Optimal couplings exist when $\mathrm{V}=\mathrm{W}$ are finite or when $\mathrm{V}=\mathrm{W}=\mathbb{R}, \rho(x, y)=(x-y)^{2}$, and both $\pi$ and $\lambda$ both have finite second moments [19].

Consider the $\left(n, R, R_{c}\right)$ randomized source code depicted in Fig. 4 which is obtained by augmenting the original $\left(n, R, R_{c}\right)$ code with the optimal coupling $T_{\hat{Y}^{n} \mid Y^{n}}$ between $\mathbf{P}_{Y^{n}}$ and $\psi^{n}$ with transportation cost $\hat{T}\left(\mathbf{P}_{Y^{n}}, \psi^{n}\right)$ when the cost function is $\rho_{n}\left(y^{n}, \tilde{y}^{n}\right)=\frac{1}{n} \sum_{i=1}^{n} \rho\left(y_{i}, \tilde{y}_{i}\right):=\frac{1}{n} \sum_{i=1}^{n} d\left(y_{i}, \tilde{y}_{i}\right)^{p}$, where $d$ is a metric on $\mathbf{Y}=\mathbf{X}$. Note that

$$
d_{n}\left(y^{n}, \tilde{y}^{n}\right):=\left(\sum_{i=1}^{n} d\left(y_{i}, \tilde{y}_{i}\right)^{p}\right)^{\frac{1}{q}},
$$

where $q=\max \{1, p\}$, defines a metric on $\mathrm{Y}^{n}$. We have

$$
\begin{aligned}
\hat{T} & \left(\mathbf{P}_{Y^{n}}, \psi^{n}\right) \\
& :=\inf \left\{\mathbb{E}\left[\rho_{n}\left(Y^{n}, \tilde{Y}^{n}\right)\right]: Y^{n} \sim \mathbf{P}_{Y^{n}}, \tilde{Y}^{n} \sim \psi^{n}\right\} \\
& =\frac{1}{n} \inf \left\{\mathbb{E}\left[\sum_{i=1}^{n} d\left(Y_{i}, \tilde{Y}_{i}\right)^{p}\right]: Y^{n} \sim \mathbf{P}_{Y^{n}}, \tilde{Y}^{n} \sim \psi^{n}\right\} \\
& =\frac{1}{n}\left(\inf \left\{\mathbb{E}\left[\sum_{i=1}^{n} d\left(Y_{i}, \tilde{Y}_{i}\right)^{p}\right]^{\frac{1}{q}}: Y^{n} \sim \mathbf{P}_{Y^{n}}, \tilde{Y}^{n} \sim \psi^{n}\right\}\right)^{q} \\
& =\frac{1}{n}\left(W_{q}\left(\mathbf{P}_{Y^{n}}, \psi^{n}\right)\right)^{q},
\end{aligned}
$$

where $W_{q}$ denotes the Wasserstein distance of order $q$ [19, Definition 6.1]. Using [19, Th. 6.15], we obtain for arbitrary fixed $y_{0}^{n} \in \mathrm{Y}^{n}$ and $r$ such that $\frac{1}{q}+\frac{1}{r}=1$,

$$
\begin{aligned}
W_{q}\left(\mathbf{P}_{Y^{n}}, \psi^{n}\right) \\
\quad \leq 2^{\frac{1}{r}}\left(\sum_{y^{n}} d_{n}\left(y_{0}^{n}, y^{n}\right)^{q}\left|\mathbf{P}_{Y^{n}}\left(y^{n}\right)-\psi^{n}\left(y^{n}\right)\right|\right)^{\frac{1}{q}} \\
\quad=2^{\frac{1}{r}}\left(\sum_{y^{n}} \sum_{i=1}^{n} \rho\left(y_{0, i}, y_{i}\right)\left|\mathbf{P}_{Y^{n}}\left(y^{n}\right)-\psi^{n}\left(y^{n}\right)\right|\right)^{\frac{1}{q}} \\
\leq 2^{\frac{1}{r}}\left(n \rho_{\max }\left\|\mathbf{P}_{Y^{n}}-\psi^{n}\right\|_{T V}\right)^{\frac{1}{q}} \\
\leq 2^{\frac{1}{r}}\left(n \rho_{\max } \exp \{-\delta n\}\right)^{\frac{1}{q}}, \text { by }(32) .
\end{aligned}
$$

Hence, we have

$$
\hat{T}_{n}\left(\mathbf{P}_{Y^{n}}, \psi^{n}\right) \leq 2^{\frac{q}{r}} \rho_{\max } \exp \{-\delta n\}
$$

Recall that $\rho(x, y)=d(x, y)^{p}$ for some $p>0$. If $p \geq 1$, then $\left\|V^{n}\right\|_{p}:=\left(E\left[\sum_{i=1}^{n}\left|V_{i}\right|^{p}\right]\right)^{1 / p}$ is a norm on $\mathbb{R}^{n}$-valued random vectors whose components have finite $p$ th moments, and if $1<p<0$, we still have $\left\|U^{n}+V^{n}\right\|_{p} \leq\left\|U^{n}\right\|_{p}+\left\|V^{n}\right\|_{p}$. Thus we can upper bound the distortion $\mathbb{E}\left[\rho_{n}\left(X^{n}, \hat{Y}^{n}\right)\right]$ of the

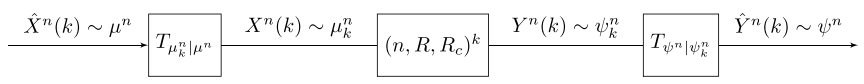

Fig. 5. Randomized source code used in the achievability proof for continuous alphabets.

code in Fig. 4 as follows:

$$
\begin{aligned}
& \left(\mathbb{E}\left[\frac{1}{n} \sum_{i=1}^{n} \rho\left(X_{i}, \hat{Y}_{i}\right)\right]\right)^{1 / q}=\left(\mathbb{E}\left[\frac{1}{n} \sum_{i=1}^{n} d\left(X_{i}, \hat{Y}_{i}\right)^{p}\right]\right)^{1 / q} \\
& \leq\left(\mathbb{E}\left[\frac{1}{n} \sum_{i=1}^{n} d\left(X_{i}, Y_{i}\right)^{p}\right]\right)^{1 / q}+\left(\mathbb{E}\left[\frac{1}{n} \sum_{i=1}^{n} d\left(Y_{i}, \hat{Y}_{i}\right)^{p}\right]\right)^{1 / q} \\
& =\left(\mathbb{E}\left[\rho_{n}\left(X^{n}, Y^{n}\right)\right]\right)^{1 / q}+\hat{T}_{n}\left(P_{Y^{n}}, \psi^{n}\right)^{1 / q},
\end{aligned}
$$

Hence, by (31) and (34) we obtain

$$
\limsup _{n \rightarrow \infty} \mathbb{E}\left[\rho_{n}\left(X^{n}, \hat{Y}^{n}\right)\right] \leq D+\delta,
$$

which completes the proof.

\section{B. Achievability for Continuous Alphabets}

In this section, we let $\mathrm{X}=\mathrm{Y}=\mathbb{R}, \rho(x, y)=(x-y)^{2}$, and assume that $\mu$ and $\psi$ have finite second moments. We make use of the discrete case to prove the achievability for the continuous case.

Assume that $\left(R, R_{C}\right)$ is in the interior of $\mathcal{L}(D)$. Then there exists $P_{X, Y, U} \in \mathcal{M}(D)$ such that $R>I(X ; U)$ and $R+R_{c}>I(Y ; U)$. Let $q_{k}$ denote the uniform quantizer on the interval $[-k, k]$ having $2^{k}$ levels, the collection of which is denoted by $L_{k}$. Extend $q_{k}$ to the entire real line by using the nearest neighborhood encoding rule. Define $X(k):=q_{k}(X)$ and $Y(k):=q_{k}(Y)$. Let $\mu_{k}$ and $\psi_{k}$ denote the distributions of $X(k)$ and $Y(k)$, respectively. It is clear that

$\mathbb{E}\left[(X-X(k))^{2}\right] \rightarrow 0$, and $\mathbb{E}\left[(Y-Y(k))^{2}\right] \rightarrow 0$ as $k \rightarrow \infty$.

Moreover, by [19, Th. 6.9] it follows that $\hat{T}\left(\mu_{k}, \mu\right) \rightarrow 0$ and $\hat{T}\left(\psi_{k}, \psi\right) \rightarrow 0$ as $k \rightarrow \infty$ since $\mu_{k} \rightarrow \mu, \psi_{k} \rightarrow \psi$ weakly [24], and $\mathbb{E}\left[X(k)^{2}\right] \rightarrow \mathbb{E}\left[X^{2}\right], \mathbb{E}\left[Y(k)^{2}\right] \rightarrow \mathbb{E}\left[Y^{2}\right]$. For each $k$ define $D_{k}:=\mathbb{E}\left[(X(k)-Y(k))^{2}\right]$. Then by (35)

$$
\lim _{k \rightarrow \infty} D_{k}=\mathbb{E}\left[(X-Y)^{2}\right] \leq D \text {. }
$$

For any $k$, let $\mathcal{M}_{k}\left(D_{k}\right)$ be the set of distributions obtained by replacing $\mu, \psi$, and $\mathrm{X}=\mathrm{Y}$ with $\mu_{k}, \psi_{k}$, and $\mathrm{X}_{k}=\mathrm{Y}_{k}=$ $L_{k}$, respectively, in (4). Note that $X(k)-U-Y(k)$ and

$$
I(X(k) ; U) \leq I(X ; U) \text { and } I(Y(k) ; U) \leq I(Y ; U)
$$

by data processing inequality which implies $R>I(X(k) ; U)$ and $R+R_{c}>I(Y(k) ; U)$. Hence, $P_{X(k), Y(k), U} \in \mathcal{M}_{k}\left(D_{k}\right)$. Then, using the achievability result for discrete alphabets, for any $k$, one can find a sequence of $\left(n, R, R_{c}\right)^{k}$ randomized source codes for common source and reproduction alphabet $L_{k}$, source distribution $\mu_{k}$, and desired output distribution $\psi_{k}$ such that the upper limit of the distortions of these codes is upper bounded by $D_{k}$.

For each $k$ and $n$, consider the randomized source codes defined in Fig. 5. We note that the definition of the optimal 
transportation cost implies that $\hat{T}\left(\mu_{k}^{n}, \mu^{n}\right) \leq \hat{T}\left(\mu_{k}, \mu\right)$ and $\hat{T}\left(\psi_{k}^{n}, \psi^{n}\right) \leq \hat{T}\left(\psi_{k}, \psi\right)$. Hence, using the triangle inequality for the norm $\left\|V^{n}\right\|_{2}:=\left(\sum_{i=1}^{n} E\left[V_{i}^{2}\right]\right)^{1 / 2}$ on $\mathbb{R}^{n}$-valued random vectors having finite second moments, for all $k$, we have

$$
\begin{aligned}
& \limsup _{n \rightarrow \infty} \mathbb{E}\left[\left(\hat{X}^{n}(k)-\hat{Y}^{n}(k)\right)^{2}\right]^{1 / 2} \\
& \leq \limsup _{n \rightarrow \infty}\left(\hat{T}\left(\mu_{k}^{n}, \mu^{n}\right)^{1 / 2}+\mathbb{E}\left[\left(X^{n}(k)-Y^{n}(k)\right)^{2}\right]^{1 / 2}\right. \\
& \left.\quad+\hat{T}\left(\psi_{k}^{n}, \psi^{n}\right)^{1 / 2}\right) \\
& \leq \hat{T}\left(\mu_{k}, \mu\right)^{1 / 2}+\hat{T}\left(\psi_{k}, \psi\right)^{1 / 2} \\
& \quad+\limsup _{n \rightarrow \infty} \mathbb{E}\left[\left(X^{n}(k)-Y^{n}(k)\right)^{2}\right]^{1 / 2} \\
& \leq \hat{T}\left(\mu_{k}, \mu\right)^{1 / 2}+\hat{T}\left(\psi_{k}, \psi\right)^{1 / 2}+D_{k}^{1 / 2} .
\end{aligned}
$$

By choosing $k$ large enough we can make the last term arbitrarily close to $D$, which completes the proof.

\section{Cardinality Bound}

In this section, we show that for any discrete distribution $\Lambda_{X, Y, W}$ forming a Markov chain $X-W-Y$, there exists a discrete distribution $\Gamma_{X, Y, U}$ forming another Markov chain $X-U-Y$ such that

$$
\begin{aligned}
|\mathrm{U}| & \leq|\mathrm{X}|+|\mathrm{Y}|+1, \\
\Gamma_{X} & =\Lambda_{X} \\
\Gamma_{Y} & =\Lambda_{Y}, \\
\mathbb{E}_{\Gamma}[\rho(X, Y)] & =\mathbb{E}_{\Lambda}[\rho(X, Y)], \\
I_{\Gamma}(X ; U) & =I_{\Lambda}(X ; W), \\
I_{\Gamma}(Y ; U) & =I_{\Lambda}(Y ; W),
\end{aligned}
$$

where $I_{P}(X ; U)$ denotes the mutual information computed with respect to the distribution $P$. Let $\mathcal{P}(\mathrm{X}) \times \mathcal{P}(\mathrm{Y})$ denote the product of probability simplices $\mathcal{P}(\mathrm{X})$ and $\mathcal{P}(\mathrm{Y})$ representing the set of all distributions of independent random variables over $\mathrm{X} \times \mathrm{Y}$. This set is compact and connected when viewed as a subset of $\mathbb{R}^{|X|+|Y|}$. Without loss of generality $\mathrm{X}=\{1, \ldots,|\mathrm{X}|\}$ and $\mathrm{Y}=\{1, \ldots,|\mathrm{Y}|\}$. Since $H(X)$ is fixed in $I(X ; W)=H(X)-H(X \mid W)$ (similarly $H(Y)$ is fixed in $I(Y ; W)=H(Y)-H(Y \mid W))$, we define the following real valued continuous functions on $\mathcal{P}(\mathrm{X}) \times \mathcal{P}(\mathrm{Y})$ :

$$
g_{j}(v)= \begin{cases}v_{x}(j), & \text { if } j=1, \ldots,|\mathrm{X}|-1 \\ v_{y}(j), & \text { if } j=|\mathrm{X}|, \ldots,|\mathrm{X}|+|\mathrm{Y}|-2 \\ \mathbb{E}_{v}[\rho(X, Y)], & \text { if } j=|\mathrm{X}|+|\mathrm{Y}|-1 \\ H\left(v_{x}\right), & \text { if } j=|\mathrm{X}|+|\mathrm{Y}| \\ H\left(v_{y}\right), & \text { if } j=|\mathrm{X}|+|\mathrm{Y}|+1,\end{cases}
$$

where $v=v_{x} \otimes v_{y}$ and $H(P)$ denotes the entropy of the distribution $P$. By so-called 'support lemma' [25, Appendix C], there exists a random variable $U \sim \Gamma_{U}$, taking values in $U$ with $|\mathrm{U}| \leq|\mathrm{X}|+|\mathrm{Y}|+1$, and a conditional probability $\Gamma_{X \mid U} \Gamma_{Y \mid U}$ on $\mathrm{X} \times \mathrm{Y}$ given $\mathrm{U}$ such that for $j=1, \ldots,|\mathrm{X}|+|\mathrm{Y}|+1$,

$$
\begin{aligned}
& \sum_{w} g_{j}\left(\Lambda_{X \mid W=w} \Lambda_{Y \mid W=w}\right) \Lambda_{W}(w) \\
& =\sum_{u} g_{j}\left(\Gamma_{X \mid U=u} \Gamma_{Y \mid U=u}\right) \Gamma_{U}(u),
\end{aligned}
$$

which completes the proof.

\section{Converse}

We use the standard approach to prove the converse in Theorem 1, i.e., that $\operatorname{cl} \mathcal{R}(D) \subset \mathcal{L}(D)$ for any $D \geq 0$. We note that this proof holds both for finite alphabets and continuous alphabets.

For each $R_{c}$, define the minimum coding rate $R$ at distortion level $D$ as

$$
\min \left\{R \in \mathcal{R}\left(D, R_{c}\right)\right\}=: I_{R_{c}}(\mu \| \psi, D) .
$$

Using a time-sharing argument and the operational meaning of $I_{R_{c}}(\mu \| \psi, D)$, one can prove that $I_{R_{c}}(\mu \| \psi, D)$ is convex in $D$, and therefore, continuous in $D, 0<D<\infty$ (see the proof of Lemma 1). Since $I_{R_{c}}(\mu \| \psi, D)$ is nonincreasing in $D$, we have $I_{R_{c}}(\mu \| \psi, 0) \geq \lim _{D \rightarrow 0} I_{R_{c}}(\mu \| \psi, D)$. But by the definition of $\mathcal{R}\left(0, R_{c}\right)$, we also have $\lim _{D \rightarrow 0} I_{R_{c}}(\mu \| \psi, D) \in$ $\mathcal{R}\left(0, R_{c}\right)$, so that $I_{R_{c}}(\mu \| \psi, 0)=\lim _{D \rightarrow 0} I_{R_{c}}(\mu \| \psi, D)$. Hence, $I_{R_{c}}(\mu \| \psi, D)$ is also continuous at $D=0$. Let us define $\mathcal{R}^{*}(D)=\left\{\left(R, R_{c}\right) \in \mathbb{R}^{2}: R>I_{R_{c}}(\mu \| \psi, D)\right\}$ and let $\left(R, R_{c}\right) \in \mathcal{R}^{*}(D)$. Since $I_{R_{c}}(\mu \| \psi, D)$ is continuous in $D$, there exists $\varepsilon>0$ such that $R>I_{R_{c}}(\mu \| \psi, D-\varepsilon)$. Hence, there exists, for all sufficiently large $n$, a $\left(n, R, R_{c}\right)$ randomized source code such that

$$
\begin{aligned}
\mathbb{E}\left[\rho_{n}\left(X^{n}, Y^{n}\right)\right] & \leq D, \\
Y^{n} & \sim \psi^{n} .
\end{aligned}
$$

For each $n$, define the random variable $Q_{n} \sim \operatorname{Unif}\{1, \ldots, n\}$ which is independent of $\left(X^{n}, Y^{n}, J, K\right)$, associated with the $n^{t h}$ randomized source code. Since $J \in\left[2^{n R}\right]$,

$$
\begin{aligned}
n R \geq H(J) \geq H(J \mid K) & \geq I\left(X^{n} ; J \mid K\right) \\
& \stackrel{(a)}{=} I\left(X^{n} ; J, K\right) \\
& =\sum_{i=1}^{n} I\left(X_{i} ; J, K \mid X^{i-1}\right) \\
& \stackrel{(b)}{=} \sum_{i=1}^{n} I\left(X_{i} ; J, K, X^{i-1}\right) \\
& \geq \sum_{i=1}^{n} I\left(X_{i} ; J, K\right) \\
& =n I\left(X_{Q_{n}} ; J, K \mid Q_{n}\right) \\
& \stackrel{(c)}{=} n I\left(X_{Q_{n}} ; J, K, Q_{n}\right),
\end{aligned}
$$

where (a) follows from the independence of $X^{n}$ and $K$, (b) follows from i.i.d. nature of the source $X^{n}$ and (c) follows from the independence of $X_{Q_{n}}$ and $Q_{n}$. Similarly, for the sum 
rate we have

$$
\begin{aligned}
n\left(R+R_{c}\right) \geq H(J, K) & \geq I\left(Y^{n} ; J, K\right) \\
& =\sum_{i=1}^{n} I\left(Y_{i} ; J, K \mid Y^{i-1}\right) \\
& \stackrel{(a)}{=} \sum_{i=1}^{n} I\left(Y_{i} ; J, K, Y^{i-1}\right) \\
& \geq \sum_{i=1}^{n} I\left(Y_{i} ; J, K\right) \\
& =n I\left(Y_{Q_{n}} ; J, K \mid Q_{n}\right) \\
& \stackrel{(b)}{=} n I\left(Y_{Q_{n}} ; J, K, Q_{n}\right),
\end{aligned}
$$

where $(a)$ follows from i.i.d. nature of the output $Y^{n}$ and (b) follows from the independence of $Y_{Q_{n}}$ and $Q_{n}$. Notice that $X_{Q_{n}} \sim \mu, Y_{Q_{n}} \sim \psi$, and $X_{Q_{n}}-\left(J, K, Q_{n}\right)-Y_{Q_{n}}$. We also have

$$
\begin{aligned}
\mathbb{E}\left[\rho\left(X_{Q_{n}}, Y_{Q_{n}}\right)\right] & =\mathbb{E}\left[\mathbb{E}\left[\rho\left(X_{Q_{n}}, Y_{Q_{n}}\right) \mid Q_{n}\right]\right] \\
& =\frac{1}{n} \sum_{i=1}^{n} \mathbb{E}\left[\rho\left(X_{Q_{n}}, Y_{Q_{n}}\right) \mid Q_{n}=i\right] \\
& =\frac{1}{n} \sum_{i=1}^{n} \mathbb{E}\left[\rho\left(X_{i}, Y_{i}\right)\right] \\
& =\mathbb{E}\left[\rho_{n}\left(X^{n}, Y^{n}\right)\right] \leq D .
\end{aligned}
$$

Define $U=\left(J, K, Q_{n}\right)$ and denote by $P_{X, Y, U}$ the distribution of $\left(X_{Q_{n}}, Y_{Q_{n}}, U\right)$. Hence, $P_{X, Y, U} \in \mathcal{M}(D)$ which implies that $\left(R, R_{c}\right) \in \mathcal{L}(D)$. Hence, $\mathcal{R}^{*}(D) \subset \mathcal{L}(D)$. But, since $\mathcal{L}(D)$ is closed in $\mathbb{R}^{2}$, we also have $\operatorname{cl} \mathcal{R}^{*}(D)=\operatorname{cl} \mathcal{R}(D) \subset \mathcal{L}(D)$.

\section{CONCLUSION}

Generalizing the practically motivated distribution preserving quantization problem, we have derived the rate distortion region for randomized source coding of a stationary and memoryless source, where the output of the code is restricted to be also stationary and memoryless with some specified distribution. For a given distortion level, the rate region consists of coding and common randomness rate pairs, where the common randomness is independent of the source and shared between the encoder and the decoder. Unlike in classical rate distortion theory, here shared independent randomness can decrease the necessary coding rate communicated between the encoder and decoder.

\section{APPENDIX}

\section{A. Proof of Lemma 1}

Let $D_{1}$ and $D_{2}$ be two distinct positive real numbers and choose $\alpha \in(0,1)$. Fix any $\varepsilon>0$. Let $\delta$ be a small positive number which will be specified later. By the definition of $I_{0}(\mu \| \psi, D)$ and by Theorem 1 there exist positive real numbers $R_{1}$ and $R_{2}$ such that

$$
R_{i} \leq I_{0}\left(\mu \| \psi, D_{i}\right)+\delta, \quad i=1,2,
$$

and such that for all sufficiently large $n$ there exist randomized $\left(n, R_{1}, 0\right)$ and $\left(n, R_{2}, 0\right)$ source codes having output distribution $\psi^{n}$ which satisfy

$$
\mathbb{E}\left[\rho_{n}\left(X^{n}, F^{(1)}\left(E^{(1)}\left(X^{n}\right)\right)\right)\right] \leq D_{1}+\delta, \quad i=1,2,
$$

where $\left(E^{(1)}, F^{(1)}\right)$ and $\left(E^{(2)}, F^{(2)}\right)$ are the encoder-decoder pairs for these codes. Let $\left\{k_{M}\right\}_{M \geq 1}$ be a sequence of positive integers such that $\lim _{M \rightarrow \infty} \frac{k_{M}}{M}=\alpha$. Let $N$ be a positive integer which will be specified later. For the source block $X^{n N}$ define the following randomized source code:

$$
\begin{aligned}
& E:=(\underbrace{E^{(1)}, \ldots, E^{(1)}}_{k_{N} \text {-times }}, \underbrace{E^{(2)}, \ldots, E^{(2)}}_{N-k_{N} \text {-times }}), \\
& F:=(\underbrace{F^{(1)}, \ldots, F^{(1)}}_{k_{N} \text {-times }}, \underbrace{F^{(2)}, \ldots, F^{(2)}}_{N-k_{N} \text {-times }}) .
\end{aligned}
$$

Note that the output distribution for this randomized source code is $\psi^{n N}$, and its rate $R$ and distortion $D$ satisfy the following

$$
\begin{aligned}
R & =\frac{1}{n N}\left(k_{N} n R_{1}+\left(N-k_{N}\right) n R_{2}\right) \\
& \leq \frac{k_{N}}{N} I_{0}\left(\mu \| \psi, D_{1}\right)+\frac{N-k_{N}}{N} I_{0}\left(\mu \| \psi, D_{2}\right)+\delta
\end{aligned}
$$

and

$$
D=\mathbb{E}\left[\rho_{n N}\left(X^{n N}, Y^{n N}\right)\right] \leq \frac{k_{N}}{N} D_{1}+\frac{N-k_{N}}{N} D_{2}+\delta .
$$

Since $\lim _{M \rightarrow \infty} \frac{k_{M}}{M}=\alpha$, one can choose $N$ and $\delta$ such that $R$ is upper bounded by $\alpha I_{0}\left(\mu \| \psi, D_{1}\right)+(1-\alpha) I_{0}\left(\mu \| \psi, D_{2}\right)+\varepsilon$ and $D$ is upper bounded by $\alpha D_{1}+(1-\alpha) D_{2}+\varepsilon$. By Definition 1 , this yields

$$
\begin{aligned}
& I_{0}\left(\mu \| \psi, \alpha D_{1}+(1-\alpha) D_{2}\right) \\
& \quad \leq \alpha I_{0}\left(\mu \| \psi, D_{1}\right)+(1-\alpha) I_{0}\left(\mu \| \psi, D_{2}\right)+\varepsilon .
\end{aligned}
$$

Since $\varepsilon$ is arbitrary, this completes the proof.

\section{B. Proof of Corollary 1}

Assume that $\left(R, R_{C}\right)$ is in the interior of $\mathcal{S}(D)$. Then there exists $P_{X, Y, U} \in \mathcal{H}(D)$ such that $R>I(X ; U)$ and $R+R_{C}>I(X, Y ; U)$. Let $\pi=P_{X, Y}$. By Theorem 2 there exists a sequence of $\left(n, R, R_{c}\right)$ randomized source codes such that

$$
\lim _{n \rightarrow \infty}\left\|P_{X^{n}, Y^{n}}-\pi^{n}\right\|=0,
$$

where $\left(X^{n}, Y^{n}\right)$ denotes the input-output of the $n^{\text {th }}$ code. Since $\rho_{n}$ is bounded, we have

$$
\begin{aligned}
\limsup _{n \rightarrow \infty} & \left|\mathbb{E}\left[\rho_{n}\left(X^{n}, Y^{n}\right)\right]-D\right| \\
= & \limsup _{n \rightarrow \infty}\left|\mathbb{E}\left[\rho_{n}\left(X^{n}, Y^{n}\right)\right]-\mathbb{E}_{\pi^{n}}\left[\rho_{n}\left(X^{n}, Y^{n}\right)\right]\right| \\
\leq & \limsup _{n \rightarrow \infty}\left\|P_{X^{n}, Y^{n}}-\pi^{n}\right\|_{T V} \rho_{\max }=0,
\end{aligned}
$$

where $\mathbb{E}_{\pi^{n}}$ denotes the expectation with respect to $\pi^{n}$. Let $T_{\hat{Y}^{n} \mid Y^{n}}$ be the optimal coupling (i.e., conditional probability) between $P_{Y^{n}}$ and $\psi^{n}$ with the transportation cost $\hat{T}\left(P_{Y^{n}}, \psi^{n}\right)$ 


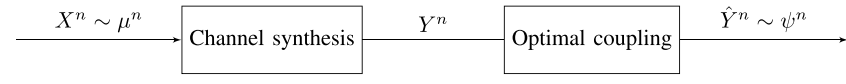

Fig. 6. Sub-optimal randomized source code achieving the rate region of Corollary 1.

with cost function $\rho_{n}$. By [19, Th. 6.15] and (37) one can prove that $\limsup _{n \rightarrow \infty} \hat{T}\left(P_{Y^{n}}, \psi^{n}\right)=0$ as in (34).

For each $n$, let us define the following encoder-decoder pair (see Fig. 6)

$$
\begin{aligned}
\tilde{E}_{J \mid X^{n}, K}^{n} & :=E_{J \mid X^{n}, K}^{n} \\
\tilde{F}_{\hat{Y}^{n} \mid J, K}^{n} & :=T_{\hat{Y}^{n} \mid Y^{n}} \circ F_{Y^{n} \mid J, K}^{n},
\end{aligned}
$$

where $\left(E^{n}, F^{n}\right)$ is the encoder-decoder pair of the $n^{\text {th }}$ code. Note that the randomized source code defined in (39) and (40) has rates $\left(R, R_{c}\right)$ and output distribution $\psi^{n}$. Furthermore, using the triangle inequality as in Section VI-A one can prove that

$$
\limsup _{n \rightarrow \infty} \mathbb{E}\left[\rho_{n}\left(X^{n}, \hat{Y}^{n}\right)\right] \leq D
$$

using (38) and the fact that $\lim _{\sup _{n \rightarrow \infty}} \hat{T}\left(P_{Y^{n}}, \psi^{n}\right)=0$. This completes the proof.

\section{Proof of Theorem 4}

Since $\mathcal{R}_{e}\left(D, R_{c}\right) \supset \mathcal{R}_{e}(D, 0)$ for all $R_{c}$, it is enough to prove that

$$
\begin{aligned}
\mathcal{R}_{e}(D, 0) & \supset \mathcal{L}(D, \infty), \\
\mathcal{R}_{e}\left(D, R_{c}\right) & \subset \mathcal{L}(D, \infty) .
\end{aligned}
$$

Recall that

$$
\mathcal{L}(D, \infty)=\left\{R \in \mathbb{R}: \exists P_{X, Y} \in \mathcal{G}(D) \text { s.t. } R \geq I(X ; Y)\right\} .
$$

Let us assume that $R \in \mathcal{L}(D, \infty)$. Then, there exists $P_{X, Y}=: \pi \in \mathcal{G}(D)$ such that $R \geq I(X ; Y)$. Fix any $\varepsilon>0$. By Theorem 3 there exists a sequence of $(n, R, \infty)$ randomized source codes such that

$$
\lim _{n \rightarrow \infty}\left\|p_{X^{n}, Y^{n}}-\pi\right\|_{T V}=0 \text { in probability, }
$$

which implies

$$
\lim _{n \rightarrow \infty}\left\|p_{Y^{n}}-\psi\right\|_{T V}=0 \text { in probability. }
$$

Hence, this sequence of codes satisfies the second constraint in Definition 3. To show that the codes satisfy the distortion constraint, we use the same steps in [21, Sec. VII-D]. We have

$$
\begin{aligned}
\rho_{n}\left(X^{n}, Y^{n}\right) & =\frac{1}{n} \sum_{i=1}^{n} \rho\left(X_{i}, Y_{i}\right) \\
& =\frac{1}{n} \sum_{i=1}^{n} \sum_{x, y} 1_{\left\{X_{i}=x, Y_{i}=y\right\}} \rho(x, y) \\
& =\sum_{x, y} \rho(x, y) \frac{1}{n} \sum_{i=1}^{n} 1_{\left\{X_{i}=x, Y_{i}=y\right\}} \\
& =\mathbb{E}_{p_{X^{n}, Y^{n}}}[\rho(X, Y)],
\end{aligned}
$$

where $1_{B}$ denotes the indicator of event $B$ and $\mathbb{E}_{p_{X^{n} Y}{ }^{n}}$ denotes the expectation with respect to the empirical distribution $p_{X^{n}, Y^{n}}$ of $\left(X^{n}, Y^{n}\right)$. For any $\varepsilon_{1}>0$, by (41) we have

$$
\operatorname{Pr}\left\{\left\|p_{X^{n}, Y^{n}}-\pi\right\|_{T V}>\varepsilon_{1}\right\}<\varepsilon_{1}
$$

for all sufficiently large $n$. Define the event $B_{\varepsilon_{1}}:=\left\{\| p_{X^{n}}\right.$, $\left.Y^{n}-\pi \|_{T V} \leq \varepsilon_{1}\right\}$. Then, for all sufficiently large $n$, we obtain

$$
\begin{aligned}
& \mathbb{E}\left[\rho_{n}\left(X^{n}, Y^{n}\right)\right] \\
& =\mathbb{E}\left[\mathbb{E}_{p_{X^{n}, Y^{n}}}[\rho(X, Y)]\right] \\
& =\mathbb{E}\left[\mathbb{E}_{p_{X^{n}, Y^{n}}}[\rho(X, Y)] 1_{B_{\varepsilon_{1}}}\right]+\mathbb{E}\left[\mathbb{E}_{p_{X^{n}, Y^{n}}}[\rho(X, Y)] 1_{B_{\varepsilon_{1}}^{c}}\right] \\
& \leq \mathbb{E}\left[\mathbb{E}_{p_{X^{n}, Y^{n}}}[\rho(X, Y)] 1_{B_{\varepsilon_{1}}}\right]+\rho_{\max } \varepsilon_{1} \\
& \leq \mathbb{E}_{\pi}[\rho(X, Y)]+2 \varepsilon_{1} \rho_{\max } \\
& \leq D+2 \varepsilon_{1} \rho_{\max } .
\end{aligned}
$$

By choosing $\varepsilon_{1}$ such that $2 \varepsilon_{1} \rho_{\max }<\varepsilon$, we obtain $\mathcal{R}_{e}(D, 0) \supset \mathcal{L}(D, \infty)$.

To prove $\mathcal{R}_{e}\left(D, R_{c}\right) \subset \mathcal{L}(D, \infty)$, we use the same arguments as in [21, Sec. VII-B]. Let us choose $R \in \mathcal{R}_{e}\left(D, R_{c}\right)$ with the corresponding sequence of $\left(n, R, R_{c}\right)$ randomized source codes satisfying constraints in Definition 3. For each $n$, define the random variable $Q_{n} \sim \operatorname{Unif}\{1, \ldots, n\}$ which is independent of the input-output $\left(X^{n}, Y^{n}\right)$ of the code $\left(n, R, R_{c}\right)$. Then, we have

$$
\begin{aligned}
n R & \geq H(J) \\
& \geq I\left(X^{n} ; Y^{n}\right) \\
& =\sum_{i=1}^{n} I\left(X_{i} ; Y^{n} \mid X^{i-1}\right) \\
& =\sum_{i=1}^{n} I\left(X_{i} ; Y^{n}, X^{i-1}\right) \\
& \geq \sum_{i=1}^{n} I\left(X_{i} ; Y_{i}\right) \\
& =n I\left(X_{Q_{n}} ; Y_{Q_{n}} \mid Q_{n}\right) \\
& \stackrel{(a)}{=} n I\left(X_{Q_{n}} ; Y_{Q_{n}}, Q_{n}\right) \\
& \geq n I\left(X_{Q_{n}} ; Y_{Q_{n}}\right),
\end{aligned}
$$

where (a) follows from the independence of $X_{Q_{n}}$ and $Q_{n}$. We also have

$$
\begin{aligned}
\mathbb{E}\left[\rho\left(X_{Q_{n}}, Y_{Q_{n}}\right)\right] & =\mathbb{E}\left[\mathbb{E}\left[\rho\left(X_{Q_{n}}, Y_{Q_{n}}\right) \mid Q_{n}\right]\right] \\
& =\frac{1}{n} \sum_{i=1}^{n} \mathbb{E}\left[\rho\left(X_{Q_{n}}, Y_{Q_{n}}\right) \mid Q_{n}=i\right] \\
& =\frac{1}{n} \sum_{i=1}^{n} \mathbb{E}\left[\rho\left(X_{i}, Y_{i}\right)\right] \\
& =\mathbb{E}\left[\rho_{n}\left(X^{n}, Y^{n}\right)\right] .
\end{aligned}
$$

One can prove $P_{Y_{Q_{n}}} \rightarrow \psi$ in total variation (see [21, Sec. VII-B-3]). Since the set of probability 
distributions over $\mathrm{X} \times \mathrm{Y}$ is compact with respect to the total variation distance, we can find a subsequence $\left\{\left(X_{Q_{n_{k}}}, Y_{Q_{n_{k}}}\right)\right\}$ of $\left\{\left(X_{Q_{n}}, Y_{Q_{n}}\right)\right\}$ such that

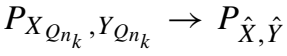

in total variation for some $P_{\hat{X}}, \hat{Y}$. But, since $P_{X_{Q_{n_{k}}}}=\mu$ for all $k$ and $P_{Y_{Q_{n}}} \rightarrow \psi$ in total variation, we must have $P_{\hat{X}}=\mu$ and $P_{\hat{Y}}=\psi$. Now, taking the limit of (42) and (43) through this subsequence, we obtain

$$
R \geq \lim _{k \rightarrow \infty} I\left(X_{Q_{n_{k}}} ; Y_{Q_{n_{k}}}\right)=I(\hat{X} ; \hat{Y})
$$

and

$$
\begin{aligned}
\mathbb{E}[\rho(\hat{X}, \hat{Y})] & =\lim _{k \rightarrow \infty} \mathbb{E}\left[\rho\left(X_{Q_{n_{k}}}, Y_{Q_{n_{k}}}\right)\right] \\
& =\lim _{k \rightarrow \infty} \mathbb{E}\left[\rho_{n_{k}}\left(X^{n_{k}}, Y^{n_{k}}\right)\right] \leq D .
\end{aligned}
$$

Hence, $R \in \mathcal{L}(D, \infty)$ which completes the proof.

\section{Proof of Theorem 5}

1) Achievability: Assume $\left(R, R_{C}\right)$ is in the interior of cl $R_{d d}(D)$. Then there exists $P_{X, Y}=: \pi \in \mathcal{G}(D)$ such that $R>I(X ; Y)$ and $R+R_{c}>H(Y)$. By [26, Th. 1] or [17, Sec. III-E], there exists a sequence of $\left(n, R, R_{c}\right)$ randomized source codes with deterministic decoder such that

$$
\left\|P_{X^{n}, Y^{n}}-\pi^{n}\right\|_{T V} \rightarrow 0 .
$$

Hence, $\left\|P_{Y^{n}}-\psi^{n}\right\|_{T V} \rightarrow 0$ and

$$
\lim _{n \rightarrow \infty} \mathbb{E}\left[\rho_{n}\left(X^{n}, Y^{n}\right)\right]=\lim _{n \rightarrow \infty} \mathbb{E}_{\pi^{n}}\left[\rho_{n}\left(X^{n}, Y^{n}\right)\right] \leq D
$$

completing the proof.

2) Converse: Let $\left(R, R_{c}\right) \in \operatorname{cl} \mathcal{R}_{d d}(D)$. Using a similar argument as in Appendix $\mathrm{C}$, one can show that

$$
n R \geq n I\left(X_{Q_{n}} ; Y_{Q_{n}}\right),
$$

and

$$
\mathbb{E}\left[\rho\left(X_{Q_{n}}, Y_{Q_{n}}\right)\right]=\mathbb{E}\left[\rho_{n}\left(X^{n}, Y^{n}\right)\right],
$$

where $Q_{n} \sim$ Unif $\{1, \ldots, n\}$ is independent of input-output $\left(X^{n}, Y^{n}\right)$ of the corresponding randomized source code, and $P_{Y_{Q_{n}}} \rightarrow \psi$ in total variation. Also, there is a subsequence

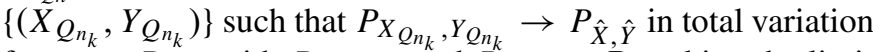
for some $P_{\hat{X}, \hat{Y}}$ with $P_{\hat{X}}=\mu$ and $P_{\hat{Y}}=\psi$. By taking the limit of (44) and (45) through this subsequence we obtain

$$
\begin{aligned}
R & \geq I(\hat{X} ; \hat{Y}), \\
\mathbb{E}[\rho(\hat{X}, \hat{Y})] & \leq D .
\end{aligned}
$$

Hence, the first inequality in (19) is satisfied. To show the second inequality, let $a_{n}:=\left\|P_{Y^{n}}-\psi^{n}\right\|_{T V}$. By [22, Th. 17.3.3], we have

$$
\left|H\left(Y^{n}\right)-H\left(\psi^{n}\right)\right| \leq a_{n} \log \left(\frac{|\mathrm{Y}|^{n}}{a_{n}}\right),
$$

where $H\left(\psi^{n}\right)=n H(\psi)$. Since the decoder is a deterministic function of $J$ and $K$, we have

$$
n H(\psi)-a_{n}\left(n \log |\mathrm{Y}|-\log a_{n}\right) \leq H\left(Y^{n}\right) \leq n\left(R+R_{c}\right) .
$$

Since $a_{n} \rightarrow 0$ as $n \rightarrow \infty$, this yields $R+R_{c} \geq H(\psi)=H(Y)$.

\section{ACKNOWLEDGMENT}

The authors would like to thank two anonymous reviewers for many constructive comments.

\section{REFERENCES}

[1] L. Roberts, "Picture coding using pseudo-random noise," IRE Trans. Inf. Theory, vol. 8, no. 2, pp. 145-154, Feb. 1962.

[2] L. Schuchman, "Dither signals and their effect on quantization noise," IEEE Trans. Commun., vol. 12, no. 4, pp. 162-165, Dec. 1964.

[3] R. M. Gray and T. G. Stockham, "Dithered quantizers," IEEE Trans. Inf. Theory, vol. 39, no. 3, pp. 805-812, May 1993.

[4] J. Ziv, "On universal quantization," IEEE Trans. Inf. Theory, vol. 31, no. 3, pp. 344-347, May 1985.

[5] R. Zamir and M. Feder, "On universal quantization by randomized uniform/lattice quantizers," IEEE Trans. Inf. Theory, vol. 38, no. 2, pp. 428-436, Mar. 1992.

[6] R. Zamir and M. Feder, "Information rates of pre/post-filtered dithered quantizers," IEEE Trans. Inf. Theory, vol. 42, no. 5, pp. 1340-1353, Sep. 1996.

[7] R. Zamir, Lattice Coding for Signals and Networks. Cambridge, U.K. Cambridge Univ. Press, 2014.

[8] E. Akyol and K. Rose, "On constrained randomized quantization," in Proc. Data Compress. Conf., Snowbird, UT, USA, Apr. 2012, pp. $72-81$.

[9] E. Akyol and K. Rose, "On constrained randomized quantization," IEEE Trans. Signal Process., vol. 61, no. 13, pp. 3291-3302, Jul. 2013.

[10] M. Li, J. Klejsa, and W. B. Kleijn, "Distribution preserving quantization with dithering and transformation," IEEE Signal Process. Lett., vol. 17, no. 12, pp. 1014-1017, Dec. 2010.

[11] M. Li, J. Klejsa, and W. B. Kleijn. (2011). "On distribution preserving quantization." [Online]. Available: http://arxiv.org/abs/1108.3728

[12] J. Klejsa, G. Zhang, M. Li, and W. B. Kleijn, "Multiple description distribution preserving quantization," IEEE Trans. Signal Process., vol. 61 no. 24, pp. 6410-6422, Dec. 2013.

[13] N. Saldi, T. Linder, and S. Yüksel, "Randomized quantization and optimal design with a marginal constraint," in Proc. IEEE Int. Symp. Inf. Theory, Jul. 2013, pp. 2349-2353.

[14] N. Saldi, T. Linder, and S. Yüksel, "Randomized quantization and source coding with constrained output distribution," IEEE Trans. Inf. Theory, vol. 61, no. 1, pp. 91-106, Jan. 2015.

[15] R. Zamir and K. Rose, "Natural type selection in adaptive lossy compression," IEEE Trans. Inf. Theory, vol. 47, no. 1, pp. 99-111, Jan. 2001

[16] P. Cuff, "Communication requirements for generating correlated random variables," in Proc. IEEE Int. Symp. Inf. Theory, Jul. 2008, pp. 1393-1397.

[17] P. Cuff, "Distributed channel synthesis," IEEE Trans. Inf. Theory, vol. 59, no. 11, pp. 7071-7096, Nov. 2013.

[18] R. M. Dudley, Real Analysis and Probability. New York, NY, USA: Chapman \& Hall, 1989.

[19] C. Villani, Optimal Transport: Old and New. New York, NY, USA: Springer-Verlag, 2009.

[20] A. D. Wyner, "The common information of two dependent random variables," IEEE Trans. Inf. Theory, vol. 21, no. 2, pp. 163-179, Mar. 1975.

[21] P. W. Cuff, H. H. Permuter, and T. M. Cover, "Coordination capacity," IEEE Trans. Inf. Theory, vol. 56, no. 9, pp. 4181-4206, Sep. 2010.

[22] T. M. Cover and J. A. Thomas, Elements of Information Theory, 2nd ed. New York, NY, USA: Wiley, 2006

[23] A. Winter. (2002). "Compression of sources of probability distributions and density operators." [Online]. available: http://arxiv.org/abs/quant$\mathrm{ph} / 0208131$

[24] P. Billingsley, Convergence of Probability Measures, 2nd ed. New York, NY, USA: Wiley, 1999.

[25] A. El Gamal and Y.-H. Kim, Network Information Theory. Cambridge, U.K.: Cambridge Univ. Press, 2011.

[26] C. Bennett, I. Devetak, A. Harrow, P. Shor, and A. Winter. (2013). "The quantum reverse Shannon theorem and resource tradeoffs for simulating quantum channels." [Online]. Available: arXiv:0912.5537v5 
Naci Saldi (S'14) received his B.Sc. and M.S. degrees in Electrical and Electronics Engineering from Bilkent University in 2008 and 2010 $\mathrm{He}$ received the $\mathrm{PhD}$ degree in the Department of Mathematics and Statistics at Queen's University in 2015 where he is currently a postdoctoral researcher. His research interests include stochastic control, source coding, and applied probability.

Tamás Linder (S'92-M'93-SM'00-F'13) received the M.S. degree in electrical engineering from the Technical University of Budapest, Hungary, in 1988, and the Ph.D degree in electrical engineering from the Hungarian Academy of Sciences in 1992.

He was a post-doctoral researcher at the University of Hawaii in 1992 and a Visiting Fulbright Scholar at the Coordinated Science Laboratory, University of Illinois at Urbana-Champaign during 1993-1994. From 1994 to 1998 he was a faculty member in the Department of Computer Science and Information Theory at the Technical University of Budapest. From 1996 to 1998 he was also a visiting research scholar in the Department of Electrical and Computer Engineering, University of California, San Diego. In 1998 he joined Queen's University where he is now a Professor of Mathematics and Engineering in the Department of Mathematics and Statistics. His research interests include communications and information theory, source coding and vector quantization, machine learning, and statistical pattern recognition.

Dr. Linder received the Premier's Research Excellence Award of the Province of Ontario in 2002 and the Chancellor's Research Award of Queen's University in 2003. He was an Associate Editor for Source Coding of the IEEE TRANSACTIONS ON INFORMATION THEORY in 2003-2004.
Serdar Yüksel (M'11) received his B.Sc. degree in Electrical and Electronics Engineering from Bilkent University in 2001; M.S. and Ph.D. degrees in Electrical and Computer Engineering from the University of Illinois at Urbana- Champaign in 2003 and 2006, respectively. He was a post-doctoral researcher at Yale University for a year before joining Queen's University as an Assistant Professor of Mathematics and Engineering in the Department of Mathematics and Statistics, where he is now an Associate Professor. He has been awarded the 2013 CAIMS/PIMS Early Career Award in Applied Mathematics. His research interests are on stochastic and decentralized control, information theory and applied probability. He is an Associate Editor for the IEEE TRAnsactions on Automatic CONTROL. 